\title{
THE KINETIC SUNYAEV-ZEL'DOVICH EFFECT AS A PROBE OF THE PHYSICS OF COSMIC REIONIZATION: THE EFFECT OF SELF-REGULATED REIONIZATION
}

\author{
Hyunbae Park $^{1}$, Paul R. Shapiro ${ }^{1}$, Eitchiro Komatsu ${ }^{1,2,3}$, Ilian T. Iliev ${ }^{4}$, Kyunguin Ahn $^{5}$, And Garrelt Mellema ${ }^{6}$ \\ ${ }^{1}$ Texas Cosmology Center and the Department of Astronomy, The University of Texas at Austin, 1 University Station, C1400, Austin, TX 78712, USA \\ ${ }^{2}$ Kavli Institute for the Physics and Mathematics of the Universe (Kavli IPMU, WPI), Todai Institutes for Advanced Study, \\ The University of Tokyo, Kashiwa, Chiba 277-8583, Japan \\ ${ }^{3}$ Max-Planck-Institut für Astrophysik, Karl-Schwarzschild Str. 1, D-85741 Garching, Germany \\ ${ }^{4}$ Astronomy Centre, Department of Physics and Astronomy, Pevensey II Building, University of Sussex, Falmer, Brighton BN1 9QH, UK \\ ${ }^{5}$ Department of Earth Science Education, Chosun University, Gwangju 501-759, Republic of Korea \\ ${ }^{6}$ Stockholm Observatory, AlbaNova University Center, Stockholm University, SE-106 91 Stockholm, Sweden \\ Received 2013 January 15; accepted 2013 April 1; published 2013 May 8
}

\begin{abstract}
We calculate the angular power spectrum of the cosmic microwave background temperature fluctuations induced by the kinetic Sunyaev-Zel'dovich (kSZ) effect from the epoch of reionization (EOR). We use detailed $\mathrm{N}$-body+radiative-transfer simulations to follow inhomogeneous reionization of the intergalactic medium. For the first time, we take into account the "self-regulation" of reionization: star formation in low-mass dwarf galaxies $\left(10^{8} M_{\odot} \lesssim M \lesssim 10^{9} M_{\odot}\right)$ or minihalos $\left(10^{5} M_{\odot} \lesssim M \lesssim 10^{8} M_{\odot}\right)$ is suppressed if these halos form in the regions that were already ionized or Lyman-Werner dissociated. Some previous work suggested that the amplitude of the kSZ power spectrum from the EOR can be described by a two-parameter family: the epoch of half-ionization and the duration of reionization. However, we argue that this picture applies only to simple forms of the reionization history which are roughly symmetric about the half-ionization epoch. In self-regulated reionization, the universe begins to be ionized early, maintains a low level of ionization for an extended period, and then finishes reionization as soon as high-mass atomically cooling halos dominate. While inclusion of self-regulation affects the amplitude of the kSZ power spectrum only modestly $(\sim 10 \%)$, it can change the duration of reionization by a factor of more than two. We conclude that the simple two-parameter family does not capture the effect of a physical, yet complex, reionization history caused by self-regulation. When added to the post-reionization kSZ contribution, our prediction for the total kSZ power spectrum is below the current upper bound from the South Pole Telescope. Therefore, the current upper bound on the kSZ effect from the EOR is consistent with our understanding of the physics of reionization.
\end{abstract}

Key words: cosmic background radiation - dark ages, reionization, first stars - early universe - galaxies: high-redshift - intergalactic medium - large-scale structure of universe - radiative transfer

Online-only material: color figures

\section{INTRODUCTION}

How was the intergalactic medium (IGM) reionized before $z=6$ ? The secondary anisotropy of the cosmic microwave background (CMB) at $l>3000$ allows us to probe the physics of cosmic reionization via the kinetic Sunyaev-Zel'dovich effect (kSZ; Sunyaev \& Zel'dovich 1980). The temperature of the $\mathrm{CMB}$ changes as free electrons in ionized gas Compton scatter CMB photons: the bulk peculiar velocity of electrons induces Doppler shifts in the energy of the CMB photons. While the spectrum of the CMB remains that of a blackbody, its temperature changes. ${ }^{7}$

Inhomogeneity in the density and velocity of electrons, as well as inhomogeneity in the ionization fraction, will induce temperature fluctuations in the CMB, $\Delta T / T$, given by

$$
\frac{\Delta T(\hat{\gamma})}{T}=-\int d \tau e^{-\tau} \frac{\hat{\gamma} \cdot \mathbf{v}}{c},
$$

where $\hat{\gamma}$ is the line-of-sight unit vector, $\mathbf{v}$ is the peculiar velocity field, and $\tau$ is the optical depth to Thomson scattering integrated

\footnotetext{
7 A related effect results from the thermal motions of free electrons in the hot intracluster gas, called the thermal SZ effect (tSZ). Multiwavelength

observations allow a distinction between the kSZ and tSZ effects on the CMB. Here, we will focus on the kSZ signal alone.
}

through the IGM from $z=0$ to the surface of last scattering at $z_{\text {rec }} \approx 10^{3}$, where

$$
d \tau=c n_{e}(z) \sigma_{\mathrm{T}}\left(\frac{d t}{d z}\right) d z
$$

There are two contributions to the kSZ signal.

1. Post-reionization contribution. This is the contribution from redshifts below $z=z_{\mathrm{ov}}$, where $z_{\mathrm{ov}}$ is the redshift at which reionization is finished, when individual $\mathrm{H}$ II bubbles fully overlap with one another. While the post-reionization contribution depends upon the value of $z_{\mathrm{ov}}$, for which quasar absorption spectra suggest $z_{\text {ov }} \sim 6-7$, it is not too sensitive to the exact value of $z_{\mathrm{ov}}$. We will not discuss this contribution in this paper, but will discuss it in a subsequent paper (H. Park et al., in preparation).

2. Reionization contribution. This is the contribution from redshifts above $z=z_{\mathrm{ov}}$, where the ionization was patchy and incomplete. This contribution depends not only on $z_{\mathrm{ov}}$, but also on the details of the time and spatial variation of inhomogeneous reionization, which are not yet well constrained; thus, we must explore how predictions vary for different models of reionization. The reionization contribution is the main focus of this paper.

Modeling the reionization contribution is a challenge, as the universe was not ionized homogeneously, but in patches. 
These patches grow over time until they overlap, finishing the reionization of the universe. The distribution of these patches is determined by nonlinear physics: nonlinear clustering of the sources of ionizing photons, nonlinear clumping of gas in the IGM, and complex morphologies of patches resulting from the propagation of ionization fronts in the clumpy IGM. Accurately calculating the reionization contribution thus requires numerical simulations of cosmological structure formation coupled with radiative transfer.

To model the formation and spatial clustering of the sources of ionizing photons, cosmological simulations must be performed in a volume large enough to capture the crucial spatial variations of this process in a statistically meaningful way. This requires a volume greater than $\sim 100$ comoving Mpc across because H II bubbles can typically grow as large as $\sim 20$ comoving Mpc in size. These simulations must also have a high enough mass resolution to resolve the formation of the individual galaxies which are the sources of ionizing radiation; thus, billions of particles are required. The radiative transfer of ionizing photons is then calculated on the IGM density and velocity fields computed by the cosmological simulation.

What do current observational data tell us? The South Pole Telescope (SPT) experiment has detected an excess temperature anisotropy for the CMB on small angular scales, which they attribute to the Sunyaev-Zel'dovich (SZ) effect. By subtracting the dominant contribution from the thermal Sunyaev-Zel'dovich (tSZ) effect by using multiwavelength observations to distinguish it from the kSZ effect, the SPT detection yields an upper limit to the total kSZ contribution. The measurements are usually reported in terms of the angular power spectrum, $C_{l} \equiv(1 / 2 l+1) \sum_{m}\left|a_{l m}\right|^{2}$. Here, $a_{l m} \equiv \int d^{2} \hat{\gamma} \Delta T(\hat{\gamma}) Y_{l m}^{*}(\hat{\gamma})$ is the coefficient of the spherical-harmonics mode, $Y_{l m}$, of $\Delta T$. The SPT Collaboration reports their measurements in terms of the quantity

$$
D_{l} \equiv \frac{l(l+1) C_{l}}{2 \pi},
$$

which we will compute in this paper. SPT has placed an upper bound on the $\mathrm{kSZ} D_{l}$ at $l=3000$ of $D_{l=3000}^{\mathrm{kSZ}}<2.8 \mu \mathrm{K}^{2}$ (Reichardt et al. 2012). The detection of the total SZ effect is complicated by the possible contamination of the fluctuating signal caused by the cosmic infrared background (CIB) from individual galaxies. The $\mathrm{kSZ}$ limit loosens to $6.7 \mu \mathrm{K}^{2}$ when an allowance is made for a possible correlation between the tSZ effect (Zel'dovich \& Sunyaev 1969) and the CIB. ${ }^{8}$ Our goal is to see whether these current upper bounds are consistent with our models of reionization.

Following the early analytical calculation done by Vishniac for linear density and velocity perturbations in a fully ionized medium (Vishniac 1987; Jaffe \& Kamionkowski 1998),

\footnotetext{
8 After our paper was written, a new paper was posted with SZ results from the Atacama Cosmology Telescope (ACT; Dunkley et al. 2013). These new results have higher uncertainty than those quoted above from SPT, but when they are combined with those of SPT and an allowance is made for a more limited tSZ-CIB correlation than that allowed by Reichardt et al. (2012), the ACT+SPT kSZ upper limit quoted by Dunkley et al. (2013) is $5.0 \mu \mathrm{K}^{2}$ (i.e., between the SPT values with and without the tSZ-CIB correlation of $6.7 \mu \mathrm{K}^{2}$ and $2.8 \mu \mathrm{K}^{2}$, respectively). Recently, Crawford et al. (2013) refined their separation of the tSZ and kSZ contributions to the total SZ power spectrum by using the tSZ bispectrum to derive the tSZ power spectrum. With an allowance for the tSZ-CIB correlation, they now report $D_{l=3000}^{\mathrm{kSZ}}=5.3_{-2.4}^{+2.2} \mu \mathrm{K}^{2}(1 \sigma$ error bars). Recently, Crawford et al. (2013) refined their separation of the tSZ and $\mathrm{kSZ}$ contribution to the total SZ power spectrum by using the tSZ bispectrum to derive the tSZ power spectrum. With an allowance for the tSZ-CIB correlation, they now report $D_{l=3000}^{\mathrm{kSZ}}=2.9 \pm 1.5 \mu \mathrm{K}^{2}(1 \sigma$ error bars), or a $95 \%$ confidence upper limit of $5.5 \mu \mathrm{K}^{2}$.
}

calculations of the kSZ effect by cosmic reionization have steadily improved over time. Further analytical calculations later incorporated the effects of inhomogeneous reionization in an approximate manner (Gruzinov \& Hu 1998; Santos et al. 2003). A "semi-numerical" approach was also developed by combining the simulated density and velocity fields from $N$-body simulations with an analytical ansatz for tracking the reionization process (Zahn et al. 2005; McQuinn et al. 2005).

Early pioneering calculations using structure formation simulations coupled with radiative transfer to model inhomogeneous reionization numerically (Gnedin \& Jaffe 2001; Salvaterra et al. 2005) underestimated the amplitude of the kSZ signal, as they used computational boxes too small to capture the impact of large-scale velocity modes and $\mathrm{H}$ in bubbles or an accurate measure of the duration of the global epoch of reionization (EOR). This was demonstrated by the first calculations of reionization based on truly large-scale ( $>100 \mathrm{Mpc}$ ) radiative-transfer simulations, which resolved the formation of all galactic halo sources above $2 \times 10^{9} M_{\odot}$ (Iliev et al. 2007b, 2008). These later simulations demonstrated the importance of a large enough simulation volume to capture the effects of long-wavelength fluctuations properly. They were also the first to realize that it is necessary to correct the kSZ power spectrum for the missing velocity power due to the finite box size of the simulations.

For the mass range of galactic halos resolved by these simulations, $\gtrsim 10^{9} M_{\odot}$, stars-the sources of reionization-were able to form when the primordial composition gas inside the halos cooled radiatively by atomic processes involving $\mathrm{H}$ atoms. They are known as "atomic-cooling halos" to distinguish them from minihalos of mass $M \lesssim 10^{8} M_{\odot}$, with virial temperature $T_{\text {vir }} \lesssim 10^{4} \mathrm{~K}$, for which star formation is possible only if $\mathrm{H}_{2}$ molecules form in sufficient abundance to cool the gas below $T_{\text {vir }}$ by rotational-vibrational line excitation. Atomic-cooling halos with $10^{8} M_{\odot} \lesssim M \lesssim 10^{9} M_{\odot}$ also exist and are even more abundant than those with $M \gtrsim 10^{9} M_{\odot}$. These low-mass atomic-cooling halos ("LMACHs"), however, are prevented from forming stars if they form within an ionized patch of the IGM where the gas pressure of the photoheated IGM opposes the accretion of baryons onto these halos. This "self-regulates" their contribution to reionization as the global ionized fraction grows with time and more and more of these halos are born within the ionized zones (Shapiro et al. 1994; Iliev et al. 2007a). While the precise value of halo mass which defines the upper edge of this "Jeans-filtered" mass range is still uncertain, the high-mass atomic-cooling halos ("HMACHs") above $\sim 10^{9} M_{\odot}$ are generally free of this suppression.

To simulate the impact of both LMACHs and HMACHs on reionization, it was necessary for Iliev et al. (2007a) to increase their halo mass resolution so as to resolve all the LMACHs, too, by reducing the simulation box size to $53 \mathrm{Mpc}$ on a side at fixed $N$-body particle number. This led to the first radiativetransfer simulations of "self-regulated" reionization, which demonstrated the importance of including and then suppressing the LMACHs to start reionization earlier and extend its duration (Iliev et al. 2007a). While the end of reionization is still set by the rapid rise of the HMACHs, in that case, when they eventually surpass the saturated contribution of the suppressible LMACHs, the effect of the LMACHs is to boost the electronscattering optical depth, $\tau$, integrated through the EOR. Such an effect can be important for the kSZ fluctuations from the EOR too, but simulating this required us to increase the simulation 
volume again while retaining the high-mass resolution required to resolve the LMACHs as well.

Our next generation of simulations involved boxes $163 \mathrm{Mpc}$ on a side, a volume large enough to predict observables like the kSZ effect, but with $N$-body simulations large enough to resolve all halos down to $10^{8} M_{\odot}$ and incorporate ionization suppression ("Jeans filtering") of the halos of mass between $10^{8} M_{\odot}$ and $10^{9} M_{\odot}$ (Iliev et al. 2012). These smaller-mass halos (LMACHs) are more abundant and likely to be more efficient ionizing sources, as they may have a higher escape fraction and emissivity (Iliev et al. 2012). However, as described above, they may be suppressed as sources if they form inside ionized regions, where ionization heats the gas and makes its pressure high enough to resist gravitational collapse into such small galaxies. Recently, an additional simulation was performed, including this new physics, in an even larger volume ( $600 \mathrm{Mpc}$; I. T. Iliev et al., in preparation).

Ahn et al. (2012) expanded the mass range even further by accounting for starlight emitted by minihalos $\left(10^{5}-10^{8} M_{\odot}\right)$ as well. In addition to their Jeans-mass filtering in ionized regions, they may also be suppressed if molecular hydrogen in minihalos is photodissociated by Lyman-Werner (LW) band photons in the UV background below $13.6 \mathrm{eV}$ also emitted by the sources of reionization. We thus have a simulated model that takes into account all the halos down to $10^{5} M_{\odot}$ as sources of reionization.

It is now important to determine if and how the $\mathrm{kSZ}$ fluctuations from the EOR are different from the previous predictions when this "self-regulated" reionization is taken into account. That is the prime focus of this paper. Some of our results were first summarized in Shapiro et al. (2012).

Recently, Mesinger et al. (2012), Zahn et al. (2012), and Battaglia et al. (2012a) compared the predicted kSZ power spectra from their semi-numerical calculations to the upper bounds from the SPT data (Reichardt et al. 2012), obtaining limits on the epoch and the duration of the reionization. Those studies concluded that for a given value of the total Thomsonscattering optical depth, the reionization contribution to the kSZ signal is mostly sensitive to the duration of the reionization defined as $\Delta z \equiv z_{99 \%}-z_{20 \%}$ (Zahn et al. 2012) or $z_{75 \%}-z_{25 \%}$ (Mesinger et al. 2012; Battaglia et al. 2012a). Zahn et al. (2012) claim that the upper bound on $D_{l=3000}^{\mathrm{kSZ}}$ from the SPT data implies $\Delta z<4(95 \% \mathrm{CL})$ for no $\mathrm{SSZ}-\mathrm{CIB}$ correlation, and $\Delta z<7(95 \%$ $\mathrm{CL})$ for the maximum possible tSZ-CIB correlation. However, as their methods are based on an analytical ansatz for the reionization process, it is necessary to use more self-consistent calculations of radiative transfer such as our simulation results to revisit this issue. We note that Zahn et al. (2011) compared their semi-numerical approach to their own numerical simulations using radiative transfer, finding an agreement at the level of $50 \%$.

The remainder of this paper is organized as follows. In Section 2, we express the kSZ power spectrum in terms of a line-of-sight integral of the transverse momentum power spectrum, and show how the transverse momentum power spectrum is related to the statistics of the density and velocity fields of ionized gas. In Section 3, we describe the details of the simulations used for our study. In Section 4, we present our predictions for the $\mathrm{kSZ}$ power spectrum and discuss the effects of inhomogeneous reionization as well as of self-regulated reionization. In Section 5, we compare our results with the recent semi-numerical calculations and show that the inclusion of self-regulated reionization qualitatively changes the parameter dependence of the kSZ power spectrum from that without selfregulation. In Section 6, we summarize our conclusions. In
Appendix A, we give the derivation of the $\mathrm{kSZ}$ power spectrum written in terms of the transverse momentum power spectrum. In Appendix B, we show how to correct for the missing power due to a finite box size of simulations in our method.

\section{BASICS}

\subsection{Angular Power Spectrum of the kSZ Effect}

As the Thomson-scattering optical depth, $\tau$, is proportional to the free electron number density, the $\mathrm{kSZ}$ effect given by Equation (1) depends mainly on the specific ionized momentum field of the ionized medium,

$$
\mathbf{q} \equiv \chi \mathbf{v}(1+\delta)
$$

henceforth referred to only as "momentum." Here, $\chi \equiv n_{e} /\left(n_{\mathrm{H}^{+}}+\right.$ $\left.2 n_{\mathrm{He}}\right)$ is the ionization fraction and $\delta \equiv(\rho-\bar{\rho}) / \bar{\rho}$ is the density contrast of baryons. In general, the baryon density is different from the dark matter density, especially on scales smaller than the Jeans length. In this paper, we will assume that baryons trace dark matter particles, as we are interested in scales bigger than the Jeans length of gas at $10^{4} \mathrm{~K}$.

We rewrite Equation (1) using $\mathbf{q}$ as

$$
\frac{\Delta T}{T}(\hat{\gamma})=-\frac{\sigma_{T} \bar{n}_{e, 0}}{c} \int \frac{d s}{a^{2}} e^{-\tau} \mathbf{q} \cdot \hat{\gamma} .
$$

Here, $\sigma_{T}$ is the Thomson-scattering cross section, $\bar{n}_{e, 0}=$ $\bar{n}_{\mathrm{H}, 0}+2 \bar{n}_{\mathrm{He}, 0}$ is the mean number density of electrons at the (fully ionized) present epoch, and $s$ is the distance traveled by photons from a source to the observer in comoving units.

The kSZ angular power spectrum is given by ${ }^{9}$ (see Appendix A for derivation; also see Ma \& Fry 2002, but note that their Equation (4) contains a typo: it is off by a factor of $\left.\left(c / H_{0}\right)^{2}\right)$ :

$$
C_{l}=\left(\frac{\sigma_{T} \bar{n}_{e, 0}}{c}\right)^{2} \int \frac{d s}{s^{2} a^{4}} e^{-2 \tau} \frac{P_{q_{\perp}}(k=l / s, s)}{2},
$$

where $\tilde{\mathbf{q}}_{\perp}(\mathbf{k})=\tilde{\mathbf{q}}(\mathbf{k})-\hat{k}[\tilde{\mathbf{q}}(\mathbf{k}) \cdot \hat{k}]$ is the projection of $\tilde{\mathbf{q}}(\mathbf{k}) \equiv$ $\int d^{3} \mathbf{x} e^{i \mathbf{k} \cdot \mathbf{x}} \mathbf{q}(\mathbf{x})$ on the plane perpendicular to the mode vector $\mathbf{k}$ (i.e., $\tilde{\mathbf{q}}_{\perp} \cdot \mathbf{k}=0$ ), $\hat{k} \equiv \mathbf{k} /|\mathbf{k}|$ is a unit vector, and $P_{q_{\perp}}$ is the power spectrum of $\tilde{\mathbf{q}}_{\perp}$ defined by $(2 \pi)^{3} P_{q_{\perp}}(k) \delta^{D}\left(\mathbf{k}-\mathbf{k}^{\prime}\right) \equiv$ $\left\langle\tilde{\mathbf{q}}_{\perp}(\mathbf{k}) \cdot \tilde{\mathbf{q}}_{\perp}^{*}\left(\mathbf{k}^{\prime}\right)\right\rangle$. Note that $\tilde{\mathbf{q}}_{\perp}$ is often called a transverse (or curl) mode. A longitudinal (or gradient) mode is parallel to $\mathbf{k}$ and is given by $\tilde{\mathbf{q}}_{\|}(\mathbf{k})=\hat{k}[\tilde{\mathbf{q}}(\mathbf{k}) \cdot \hat{k}]$.

As we show in Appendix A, in the small-angle approximation, the line-of-sight integral cancels out the contribution from $\tilde{\mathbf{q}}_{\|}$ and half of the power of $\tilde{\mathbf{q}}_{\perp}$, leaving only the remaining half of $P_{q_{\perp}}$. This explains a factor of two in the denominator of Equation (6).

Helium atoms are assumed to be singly ionized where hydrogen atoms are ionized at least until $z_{\text {ov }}$, the redshift at which all the HiI bubbles overlap to finish the ionization of hydrogen atoms due to the similar ionization potential of $\mathrm{H} \mathrm{I}$

\footnotetext{
9 All previous numerical calculations of the kSZ power spectrum first created maps using Equation (5) and then measured $C_{l}$ from the two-dimensional Fourier transform of the simulated maps. In this paper, we will use Equation (6) to compute $C_{l}$ using $P_{q \perp}$ measured from three-dimensional simulation boxes at various redshifts without ever creating maps. While we are the first to apply this method to the computation of the kSZ power spectrum, this method has been applied successfully to the computation of the tSZ power spectrum (Refregier et al. 2000) as well as to that of the power spectrum of anisotropy of the near-infrared background (Fernandez et al. 2010, 2012).
} 


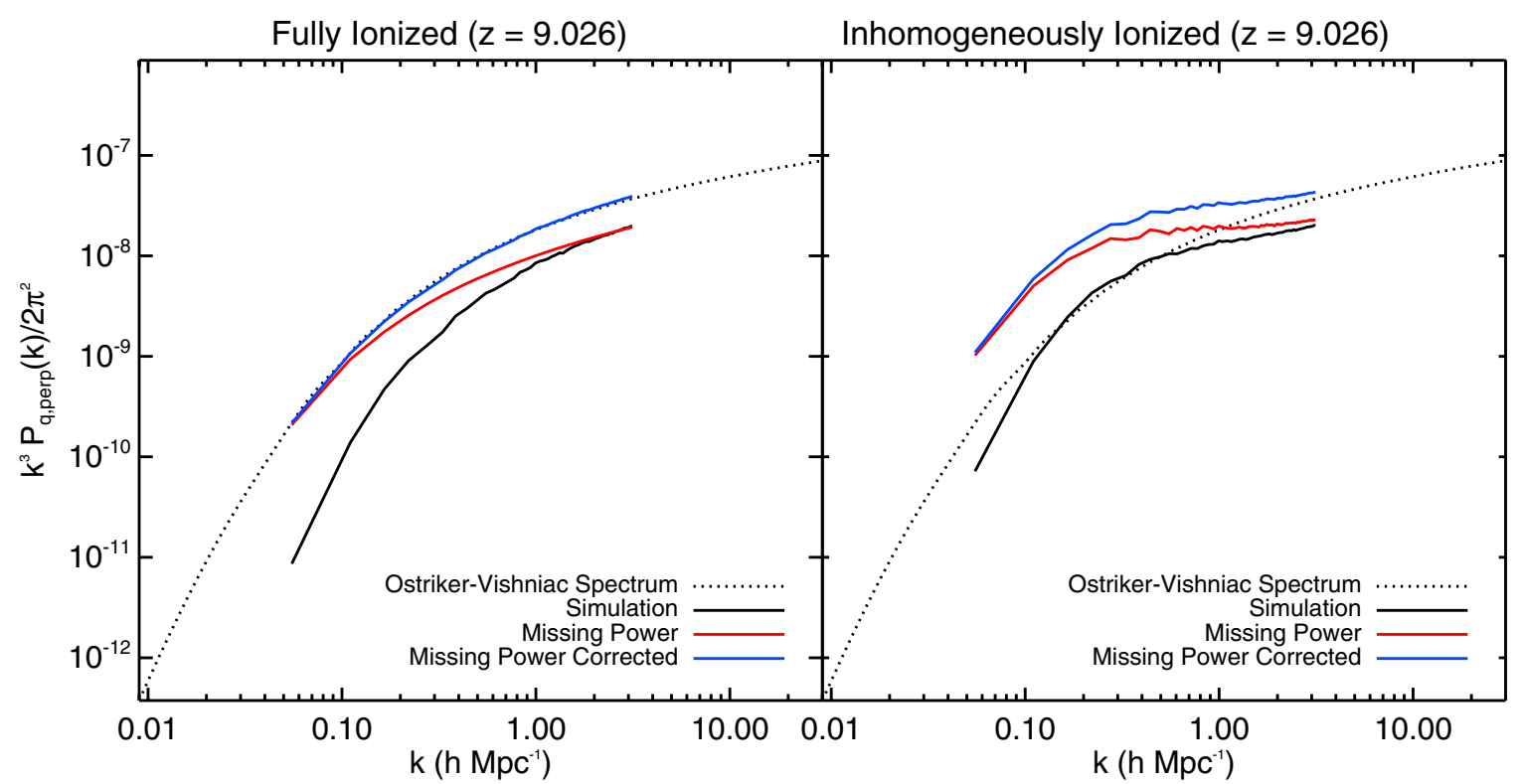

Figure 1. Dimensionless power spectra of the curl of the momentum field, $k^{3} P_{q \perp}(k) /\left(2 \pi^{2}\right)$, at $z=9$ calculated from the simulation with $114 h^{-1} \mathrm{Mpc}$ in a side. The black solid lines show the raw power spectrum obtained from the $N$-body simulation, while the blue lines show the power spectrum after being corrected for the missing velocity power due to a finite box size of the simulation. The red lines show the missing power added to the black solid lines. The dotted lines show the analytical OV spectrum given in Equation (8). Left: fully ionized case. An excellent agreement between the OV spectrum and the corrected power spectrum shows the validity of our simulation as well as that of our method to correct for the missing velocity power. Right: inhomogeneously ionized case, L3. The power spectrum is significantly enhanced at $k \lesssim 1 h \mathrm{Mpc}^{-1}$.

(A color version of this figure is available in the online journal.)

and He I. Helium atoms remain singly ionized until much later, $z \approx 3$, after which they are thought to be doubly ionized. As we are interested only in the epoch of hydrogen reionization, $z \gtrsim 6$, we will assume that the ionized fraction, $\chi$, is given by $\chi=(0.92) X$, where $X$ is the hydrogen ionized fraction at each point in our radiative-transfer simulation: $\chi$ saturates at 0.92 in fully ionized regions during hydrogen reionization, as $8 \%$ of the electrons are left bound in singly ionized helium atoms.

\subsection{Power Spectrum of the Curl of the Momentum}

Our goal is to compute the power spectrum of the curl of the momentum field, $P_{q_{\perp}}$, and evaluate Equation (6) to obtain $C_{l}$.

Assuming that the velocity field stays longitudinal, i.e., parallel to $\mathbf{k}, P_{q_{\perp}}$ is given by the second-order term in the momentum: $\mathbf{q}_{\perp}=\left(\int\left(d^{3} k^{\prime} /(2 \pi)^{3}\right) \delta\left(\mathbf{k}-\mathbf{k}^{\prime}\right) \mathbf{v}\left(\mathbf{k}^{\prime}\right)\right)_{\perp}$. This assumption is exact in the linear regime and is approximately true in the nonlinear regime, as this second-order term dominates in the nonlinear regime anyway. This gives (Ma \& Fry 2002)

$$
\begin{aligned}
P_{q_{\perp}}(k, z)= & \int \frac{d^{3} k^{\prime}}{(2 \pi)^{3}}\left(1-\mu^{\prime 2}\right)\left[P_{\delta \delta}\left(\left|\mathbf{k}-\mathbf{k}^{\prime}\right|\right) P_{v v}\left(k^{\prime}\right)\right. \\
& \left.-\frac{k^{\prime}}{\left|\mathbf{k}-\mathbf{k}^{\prime}\right|} P_{\delta v}\left(\left|\mathbf{k}-\mathbf{k}^{\prime}\right|\right) P_{\delta v}\left(k^{\prime}\right)\right]
\end{aligned}
$$

where $\mu^{\prime} \equiv \hat{k} \cdot \hat{k}^{\prime}$. Here, the $P_{\delta \delta} P_{v v}$ term gives a positive contribution, whereas the $P_{\delta v} P_{\delta v}$ term gives a negative contribution from the density field correlated with the velocity field that does not have a curl component.

Due to the finite box size of the simulations, we must correct for the missing velocity power coming from modes whose wavelength is longer than the size of the simulation box (Iliev et al. 2007b). We will describe our correction method in Appendix B.

At high redshift where the density and velocity fields are still in the linear regime, the velocity power spectrum is related to the linear density power spectrum by $P_{v v}(k)=(\dot{a} f / k)^{2} P_{\delta \delta}^{\operatorname{lin}}(k)$, where $f \equiv d \ln \delta / d \ln a$ and $a(t)$ is the Robertson-Walker scale factor. This gives the so-called Ostriker-Vishniac (OV) spectrum (Vishniac 1987):

$$
\begin{aligned}
P_{q_{\perp}}^{\mathrm{OV}}(k, z)= & \dot{a}^{2} f^{2} \int \frac{d^{3} k^{\prime}}{(2 \pi)^{3}} P_{\delta \delta}^{\operatorname{lin}}\left(\left|\mathbf{k}-\mathbf{k}^{\prime}\right|, z\right) P_{\delta \delta}^{\operatorname{lin}}\left(k^{\prime}, z\right) \\
& \times \frac{k\left(k-2 k^{\prime} \mu^{\prime}\right)\left(1-\mu^{\prime 2}\right)}{k^{\prime 2}\left(k^{2}+k^{\prime}-2 k k^{\prime} \mu^{\prime}\right)} .
\end{aligned}
$$

The OV spectrum provides a useful check of the numerical simulation and the way we correct for the missing velocity. In the left panel of Figure 1, we show an excellent agreement between the OV spectrum and the simulation result at $z=9$, after correcting for the missing velocity power due to the finite box size of the simulation.

Finally, one can incorporate the effect of inhomogeneous reionization into the equation by replacing $\delta$ in Equation (7) by $\chi(1+\delta)$ :

$$
\begin{aligned}
P_{q_{\perp}}(k, z)= & \int \frac{d^{3} k^{\prime}}{(2 \pi)^{3}}\left(1-\mu^{\prime 2}\right)\left[P_{\chi(1+\delta), \chi(1+\delta)}\left(\left|\mathbf{k}-\mathbf{k}^{\prime}\right|\right) P_{v v}\left(k^{\prime}\right)\right. \\
& \left.-\frac{k^{\prime}}{\left|\mathbf{k}-\mathbf{k}^{\prime}\right|} P_{\chi(1+\delta), v}\left(\left|\mathbf{k}-\mathbf{k}^{\prime}\right|\right) P_{\chi(1+\delta), v}\left(k^{\prime}\right)\right] .
\end{aligned}
$$

Note that we do not use this equation to compute $P_{q_{\perp}}$, but compute $P_{q_{\perp}}$ directly from the simulation. However, we use this equation to estimate and correct for the missing power due to the finite box size of the simulation as described in Appendix B. We then use the corrected $P_{q_{\perp}}$ in Equation (6) to compute the angular power spectrum. As shown in the right panel of Figure 1, the effect of reionization inhomogeneity substantially boosts the power spectrum relative to the homogeneously ionized case, while correcting for the missing velocity power of the finite simulation volume boosts it even further. 


\section{REIONIZATION SIMULATION}

\subsection{Basic Simulation Parameters}

The simulations that we will use in this paper consist of two parts: (1) cosmological $N$-body simulations of collisionless particles using the "CubeP ${ }^{3} \mathrm{M}$ " $N$-body code (Harnois-Deraps et al. 2012) and (2) radiative transfer of $\mathrm{H}$-ionizing photons in the density and source fields created from the $N$-body simulation results using the " $\mathrm{C}^{2}$-Ray" (Conservative, Causal Ray tracing) code (Mellema et al. 2006). The details of the simulations that we will use in this paper are described in Iliev et al. (2012) and Ahn et al. (2012).

Unless specified otherwise, the reionization simulations are run on the density and source fields from the same $N$-body results with $3072^{3}$ particles in a comoving box of $114 h^{-1} \mathrm{Mpc}$ on a side. Halos are identified down to $10^{8} M_{\odot}$ with at least 20 particles, using a spherical overdensity halo finder with an overdensity of 178 times the mean cosmic density. One of the models uses another $N$-body simulation with a larger box of $425 h^{-1} \mathrm{Mpc}$, with $5488^{3}$ particles, resolving halos down to $10^{9} M_{\odot}$. The background cosmology is based on the Wilkinson Microwave Anisotropy Probe 5-year data combined with constraints from baryonic acoustic oscillations and highredshift Type Ia supernovae $\left(\Omega_{M}=0.27, \Omega_{\Lambda}=0.73, h=\right.$ $0.7, \Omega_{b}=0.044, \sigma_{8}=0.8, n_{s}=0.96$; Komatsu et al. 2009).

For the $114 h^{-1} \mathrm{Mpc}$, we then calculate the IGM density field from the particle data with the halos excluded adaptively smoothed on to a $256^{3}$ radiative-transfer grid in order to generate ionization maps using the $\mathrm{C}^{2}$-Ray code. Therefore, the final physical length resolution of the reionization models is $d_{\text {cell }}=0.45 h^{-1} \mathrm{Mpc}$. The highest $l$-mode that we can calculate from the simulation is given by $l_{\text {limit }}=k_{\mathrm{Nyq}} s\left(z_{\mathrm{ov}}\right)$, where $k_{\mathrm{Nyq}}=\pi /\left(2 d_{\text {cell }}\right)$ is the Nyquist frequency and $s\left(z_{\mathrm{ov}}\right)$ is the comoving distance out to the end of reionization. For example, $z_{\mathrm{ov}}=6.6$ gives $l_{\text {limit }}=22,000$.

The new simulations also incorporate the effects of even smaller halos in $10^{5} M_{\odot}<M<10^{8} M_{\odot}$, using a sub-grid prescription calibrated by smaller-box $N$-body simulations with higher resolution having $1728^{3}$ particles in a box of $6.3 h^{-1} \mathrm{Mpc}$ (Ahn et al. 2012). Specifically, we find that there is a correlation between the number of these small-mass halos in each cell and the total matter density averaged over that cell, with cells of size $0.45 h^{-1} \mathrm{Mpc}$, which coincides with the size of the radiativetransfer cells in our $114 h^{-1} \mathrm{Mpc} \mathrm{C}^{2}$-Ray simulations. We then use this correlation to calculate the number of small-mass halos in each of the radiative-transfer cells in our $114 h^{-1} \mathrm{Mpc}$ simulations.

For our most recent simulation, in a box $425 h^{-1} \mathrm{Mpc}$ on a side, the RT grid has $504^{3}$ cells, so $d_{\text {cell }}=0.84 h^{-1} \mathrm{Mpc}$, slightly larger than that for the other simulations, and $l_{\text {limit }} \sim 12,000$. In this larger-box simulation, low-mass halos between $10^{8}$ and $10^{9} M_{\odot}$ are included by a sub-grid model like that described above for minihalos (MHs).

\subsection{Varying Physics of Reionization}

What kind of sources are responsible for reionization? In this section, we consider a set of reionization simulations based on source models of increasing sophistication from the one with only high-mass sources to the one with all kinds of sources down to the least massive halos in our models.

For each halo identified in our simulation, we calculate the number of ionizing photons that escape from it into the IGM per unit time, $\dot{N}_{\gamma}$, which is assumed to be proportional to the halo mass, $M$ :

$$
\dot{N}_{\gamma}=\frac{f_{\gamma} M \Omega_{b}}{\Delta t \Omega_{0} m_{p}},
$$

where $m_{p}$ is the proton mass, $\Delta t$ is the duration of each starforming episode (i.e., which corresponds in practice to the radiative-transfer simulation time step), and $f_{\gamma}=f_{\text {esc }} f_{\star} N_{\star}$ is the number of ionizing photons produced and released by the halo over the lifetime of the stars that form inside it in this time step, per halo atom, if $f_{*}$ is the fraction of the halo atoms that form stars during this burst, $f_{\text {esc }}$ is the fraction of the ionizing photons produced by these stars that escapes into the IGM, and the integrated number of ionizing photons released over their lifetime per stellar atom is given by $N_{\star}$. The latter parameter depends on the assumed initial mass function (IMF) for the stellar population and can range from $\sim 4000$ (e.g., for Pop II stars with a Salpeter IMF) to $\sim 100,000$ (e.g., for a top-heavy IMF of Pop III stars). Halos were assigned different efficiencies according to their mass and grouped according to whether their mass was above ("HMACHs") or below ("LMACHs") $10^{9} M_{\odot}$ (but above $10^{8} M_{\odot}$, the minimum resolved halo mass). Lowmass sources are assumed to be suppressed within ionized regions (for ionization fraction higher than 10\%) through Jeansmass filtering, as discussed in Iliev et al. (2007a).

In addition to the source efficiency parameter, $f_{\gamma}$, we also define a slightly different factor, $g_{\gamma}$, that is given by

$$
g_{\gamma}=f_{\gamma}\left(\frac{10 \mathrm{Myr}}{\Delta t}\right),
$$

where $\Delta t$ is the time between two snapshots from the $N$-body simulation. The new factor $g_{\gamma}$ reflects the fact that a given halo has a luminosity that depends on the ratio of $f_{\gamma}$ to $\Delta t$, so $g_{\gamma}$ has the advantage that it is independent of the length of the time interval between the density slices, and as such it allows a direct comparison between runs with different $\Delta t$. For the reader's convenience, we listed the values of both parameters in Table 1. The specific numerical values of the efficiency parameters are strongly dependent on the background cosmology adopted and the minimum source halo mass. Therefore, parameter values for simulations based on different underlying cosmology and halo mass resolution should not be compared directly, but require cosmology and resolution-dependent conversion coefficients to achieve the same reionization history.

\subsubsection{HMACHs-only Model}

In our simplest model (labeled L3; see Table 1 for the details; note that "L" stands for a "large volume"), we only use HMACHs as the sources of reionization. These sources are defined as the halos with $M>2.2 \times 10^{9} M_{\odot}$ for L3 and with $M>10^{9} M_{\odot}$ (corresponding to $T_{\text {vir }} \gtrsim 8 \times 10^{4} \mathrm{~K}$ at $z=9$ from the Truncated Isothermal Sphere model of Iliev \& Shapiro 2001) for the other configurations. These sources are believed to form stars even when immersed in ionized regions, due to the fact that their gravitational potential wells are deep enough to overcome Jeansmass filtering.

\subsubsection{HMACHs+LMACHs Models}

What about smaller-mass halos? LMACHs are more abundant; however, if they form inside the regions that have already been ionized, then they would not act as sources of ionizing photons. This is because ionization heats the gas and makes its pressure too high for the gas to collapse into such small halos (Iliev et al. 2007a and references therein). 
Table 1

Reionization Simulation Parameters and Global Reionization History Results

\begin{tabular}{|c|c|c|c|c|c|c|c|c|}
\hline Label & Sources & $g_{\gamma, \mathrm{H}}\left(f_{\gamma, \mathrm{H}}\right)$ & $g_{\gamma, \mathrm{L}}\left(f_{\gamma, \mathrm{L}}\right)$ & $g_{\gamma, \mathrm{MH}}\left(f_{\gamma, \mathrm{MH}}\right)^{\mathrm{a}}$ & $\tau_{\mathrm{es}}$ & $z_{10 \%}$ & $z 90 \%$ & $z_{\mathrm{ov}}$ \\
\hline L1 & HMACHs+LMACHs & $8.7(10)$ & $130(150)$ & $\cdots$ & 0.080 & 13.3 & 8.6 & 8.3 \\
\hline L2(XL2) & HMACHs+LMACHs & $1.7(2)$ & $8.7(10)$ & $\cdots$ & 0.058 & 9.9 & 6.9 & 6.8 \\
\hline L2M1J1 & HMACHs+LMACHs+MHs & $1.7(2)$ & $8.7(10)$ & $5063(1030)$ & 0.086 & 17.4 & 6.9 & 6.8 \\
\hline L3 & HMACHs only & $21.7(25)$ & $\cdots$ & $\cdots$ & 0.070 & 10.3 & 9.1 & 8.4 \\
\hline
\end{tabular}

Note. ${ }^{\text {a }} \mathrm{MH}$ efficiencies $g_{\gamma, \mathrm{MH}}\left(f_{\gamma, \mathrm{MH}}\right)$ quoted here are for the minimum-mass halo assumed to contribute, $10^{5} M_{\odot}$, which is roughly comparable to the average value for the minihalos integrated over the halo mass function. The efficiency of any MH of a given mass $M$ is obtained simply by multiplying to the quoted $g_{\gamma, \mathrm{MH}}\left(f_{\gamma, \mathrm{MH}}\right)$ by $\left(10^{5} M_{\odot} / M\right)$.

When we include LMACHs and account for this "selfregulation" of reionization, we give LMACHs a higher efficiency, $g_{\gamma}$, than for HMACHs, as presumably it is easier for ionizing photons to escape from LMACHs than from HMACHs, and Pop III stars with a top-heavy IMF, which are capable of producing more ionizing photons than Pop II stars with a Salpeter IMF, are more likely to form in LMACHs. If HMACHs are formed by mergers of smaller-mass halos, for example, they are more likely to have enough metallicity to make the transition from Pop III to Pop II star formation, and hence to a less efficient IMF.

There are two cases that have both HMACHs and LMACHs, and we will call them L1 and L2. For L1, the efficiency parameter, $g_{\gamma}$, is chosen such that the overlap in redshift, $z_{\mathrm{ov}}=8.3$, is similar to that of $\mathrm{L} 3, z_{\mathrm{ov}}=8.4$ (see Table 1 ). For $\mathrm{L} 2, g_{\gamma}$ is chosen such that $z_{\mathrm{ov}}$ is between 6 and 7 , as suggested by the quasar absorption line observations.

For L2, we have another run with a much larger volume $\left(425 h^{-1} \mathrm{Mpc}\right)$ with $504^{3}$ of radiative-transfer grids. Although it does not resolve LMACHs, we include LMACHs as a subgrid model using the correlation between the average density of radiative-transfer cells and the number density of LMACHs in a manner similar to how Ahn et al. (2012) included MHs in the simulation (I. T. Iliev et al., in preparation and K. Ahn et al., in preparation). This run gives $l_{\text {limit }} \sim 12,000$. We will call this configuration "XL2," as the volume for this run is bigger (hence the name, XL) than those runs with "L." This run will be used to check our method to correct for the missing velocity power.

\subsubsection{HMACHs+LMACHs+MHs Model}

What about even smaller-mass sources? Gas in halos of masses between $10^{5} M_{\odot}$ and $10^{8} M_{\odot}$ is thought to cool via rotational and vibrational transitions of hydrogen molecules and form stars until hydrogen molecules are dissociated by LW photons in the UV background from other sources (see Ahn et al. 2012 and references therein).

The MHs form earlier than LMACHs or HMACHs, and thus can start reionization of the universe earlier. However, as the star formation in MHs is vulnerable to LW photons, it is suppressed wherever the intensity of the LW background rises above the threshold for suppression, locally at first, and eventually globally. This adds another kind of "self-regulation" to the reionization history, with an even more extended phase of low-level ionization before MHs are eventually suppressed completely (Ahn et al. 2012).

The effects of MHs have been added to L2 by Ahn et al. (2012), and we take one of the cases simulated there, L2M1J1, as our fiducial case with MHs. See Table 1 for the efficiency of MHs. "M" denotes the mass spectrum of Pop III stars in MHs and " $\mathrm{J}$ " is the threshold intensity of the LW photon background above which the star formation in MHs is suppressed. In L2M1J1, each halo is assumed to host one Pop III star with a mass of $300 M_{\odot}$, and the assumed LW threshold is $J_{\mathrm{LW} \text {,th }}=$ $10^{-22} \mathrm{erg}^{-1} \mathrm{~cm}^{-2} \mathrm{sr}^{-1}$.

This parameter choice for $M_{*}$ and $J_{\mathrm{LW}, \text { th }}$ is only illustrative. As we discussed in Ahn et al. (2012), the nature of the selfregulated suppression of $\mathrm{MH}$ star formation is such that the contribution of $\mathrm{MH}$ stars to reionization rises to the point at which the global mean LW intensity reaches the threshold value for suppression. As long as MH stars dominate reionization (i.e., early phase), they continue to form at the global rate necessary to keep $J_{\mathrm{LW}}$ at this level, regardless of the value of $M_{*}$. For $M_{*} \gtrsim$ $100 M_{\odot}$, the ratio of ionizing to dissociating photons emitted per $\mathrm{MH}$ star is fixed, so their early contribution to reionization is also fixed by this self-regulation effect. Eventually, the LMACH and HMACH populations grow to dominate the LW background and suppress star formation inside MHs completely thereafter. The value adopted for $J_{\mathrm{LW}}$,th only somewhat affects the transition redshift at which this occurs (i.e., higher values allow MHs to contribute longer). In short, the reionization history is relatively insensitive to the value adopted for $M_{*}$ if $M_{*} \gtrsim 100 M_{\odot}$, but is somewhat more sensitive to $J_{\mathrm{LW}}$,th . The recent suggestion that MH stars may form with lower values of $M_{*}$ (e.g., $40 M_{\odot}$ ), perhaps with more than one star at a time, may alter some of these details, but the qualitative effect of self-regulation should remain. Similarly, the effect of a relative drift velocity between dark matter and baryons identified by Tseliakhovich \& Hirata (2010), which tends to raise the minimum mass of MHs which typically form stars, is offset by a small shift in the timing of the early phase of $\mathrm{MH}$-dominated reionization, as the exponential rise of $\mathrm{MH}$ abundances compensates at lower redshift.

\section{RESULTS}

Before presenting and discussing our predictions for the $\mathrm{kSZ}$ power spectrum, let us briefly comment on the global ionization history of the universe, which is the key to understanding the difference between our results and the previous ones. For more detailed discussion on the effects of self-regulation, see Iliev et al. (2007a, 2012) and Ahn et al. (2012).

Figure 2 shows how reionization proceeds in our simulation boxes, while Figure 3 shows the mass-averaged ionization fraction of the universe as a function of redshift. Both figures show that the inclusion of low-mass halos (LMACHs and MHs), which are self-regulated, significantly extends the ionization history of the universe toward higher redshift. Let us compare $\mathrm{L} 1$ and L3. As LMACHs form earlier, the universe begins to be ionized earlier in L1 than in L3. However, the universe does not get reionized quickly but keeps a low level of ionization for an extended period due to self-regulation of sources. Only 


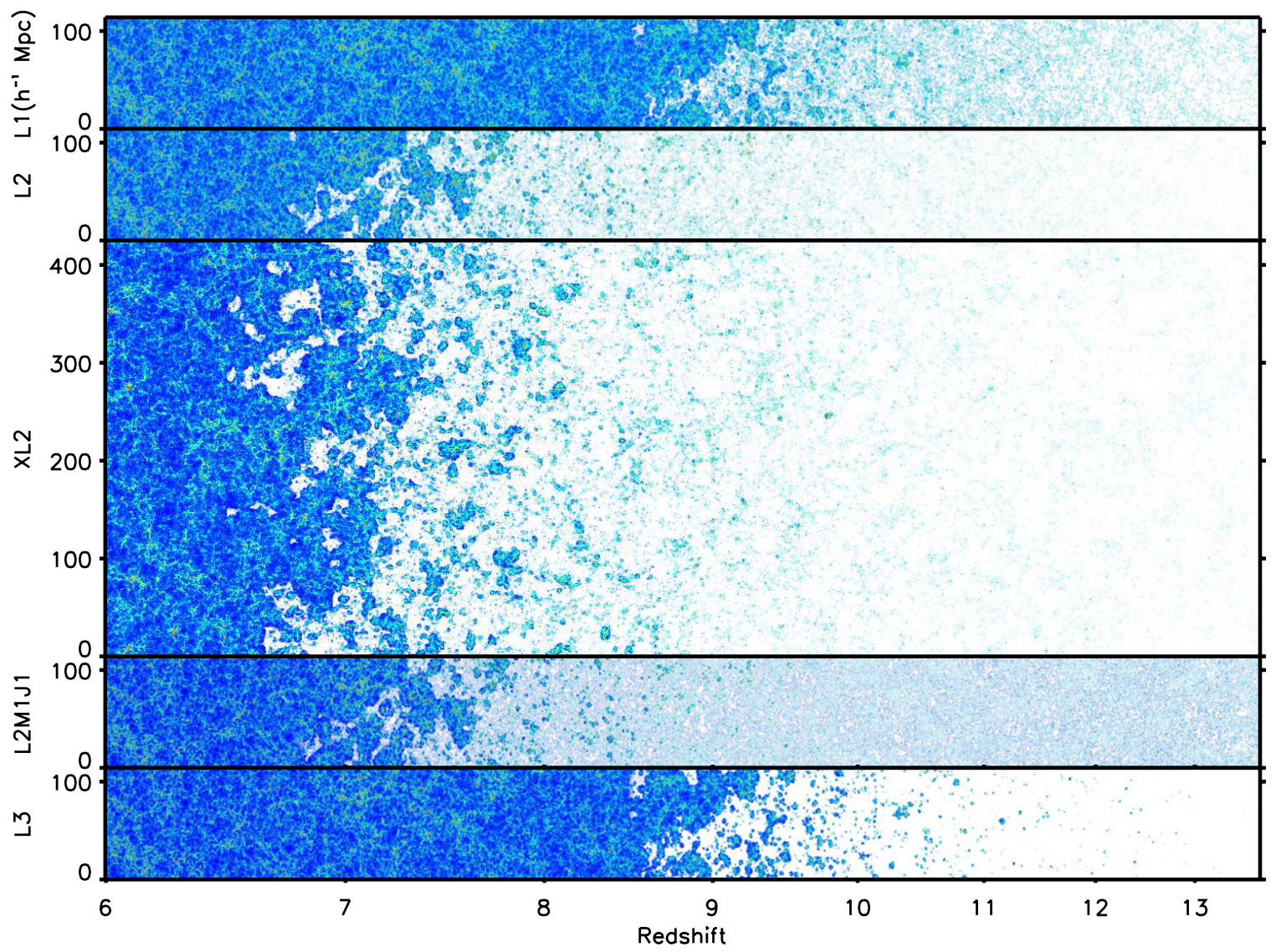

Figure 2. Cuts through the $N$-body+radiative-transfer simulations used in this work. See Table 1 for the parameters of models L1, L2, L2M1J1, and L3. While these runs have a box size of $114 h^{-1} \mathrm{Mpc}$, model XL2 has a box size of $425 \mathrm{~h}^{-1} \mathrm{Mpc}$ and has the same model parameters as model L2. Each panel shows the matter density distribution multiplied by spatially varying ionization fractions. For example, it just shows the matter density when a given region is fully ionized, while it shows nothing (i.e., white) when a given region is fully neutral. The density fields are color-coded such that overdense regions are red and underdense regions are blue. We create this figure by interpolating between adjacent snapshots at a given look-back time. The length scale is linear in the comoving units. The $x$-axis shows redshifts, while the $y$-axis shows $h^{-1} \mathrm{Mpc}$.

(A color version of this figure is available in the online journal.)

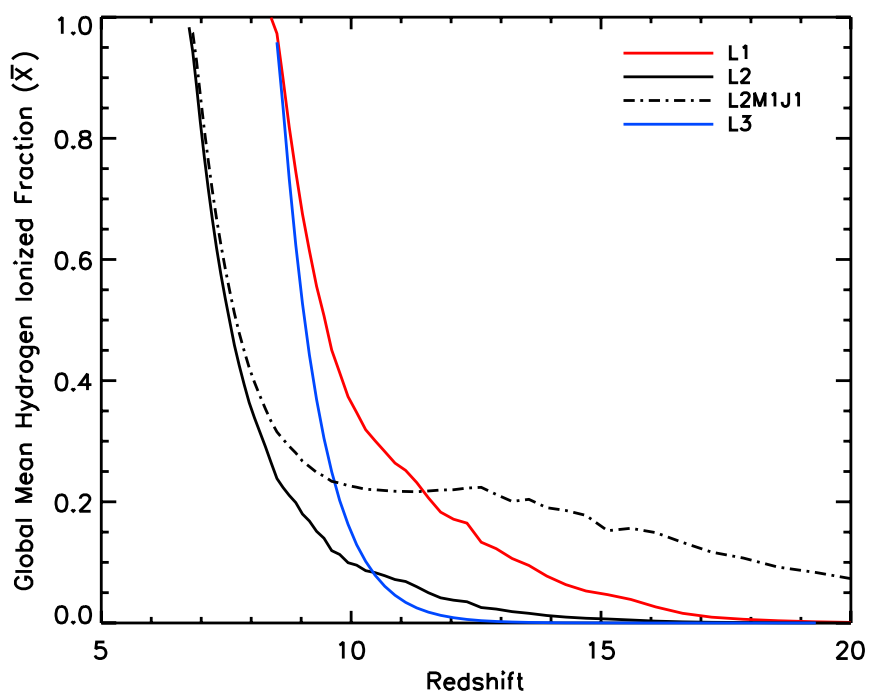

Figure 3. Global mean ionization history of our models (see Table 1 for the parameters of models). The mass-averaged hydrogen ionization fraction, $\bar{X}$, is plotted against $z$. Note how self-regulation results in an extended period of lowlevel ionization by comparing the case without self-regulation ( $\mathrm{L} 3=\mathrm{HMACHs}$ only) and that with self-regulation (L1 = HMACHs + LMACHs; Iliev et al. 2012). A further extension occurs when MH sources are included as well (i.e., compare $\mathrm{L} 2=\mathrm{HMACHs}+\mathrm{LMACHs}$ and $\mathrm{L} 2 \mathrm{M} 1 \mathrm{~J} 1=\mathrm{L} 2+\mathrm{MHs}$; Ahn et al. 2012).

(A color version of this figure is available in the online journal.) after HMACHs start to dominate at $z \sim 10$ does reionization proceed rapidly and finish soon after. In L3, with no LMACHs, by contrast, reionization proceeds rapidly from beginning to end because the abundance of HMACHs, the only sources, grows exponentially without any suppression effects to self-regulate them. When MHs are included (L2M1J2), the universe begins to ionize even earlier than the cases with HMACHs and LMACHs, and keeps a low-level ionization for a longer period.

These physically motivated yet somewhat complex reionization histories were not considered in any of the previous calculations of the kSZ power spectrum. In this section, we show that it is these new features in the reionization history that invalidate simple two-parameter descriptions of the amplitude of the kSZ power spectrum proposed by the previous study (Zahn et al. 2012; Mesinger et al. 2012; Battaglia et al. 2012a).

\subsection{Impact of Inhomogeneous Reionization}

First, it is useful to understand how important it is to include the inhomogeneity (or patchiness) of reionization when computing the kSZ power spectrum. In order to see this, we create a homogeneous version of L3 ("L3-homogeneous") in which we wipe out the inhomogeneity of reionization by replacing the ionization fraction, $\chi$, with its global average, $\bar{\chi}$ (see Figure 3). This then gives the transverse momentum power spectrum as $P_{q_{\perp}}=\bar{\chi}^{2} P_{q_{\perp}}^{\mathrm{OV}}$, where $P_{q_{\perp}}^{\mathrm{OV}}$ is the $\mathrm{OV}$ spectrum 


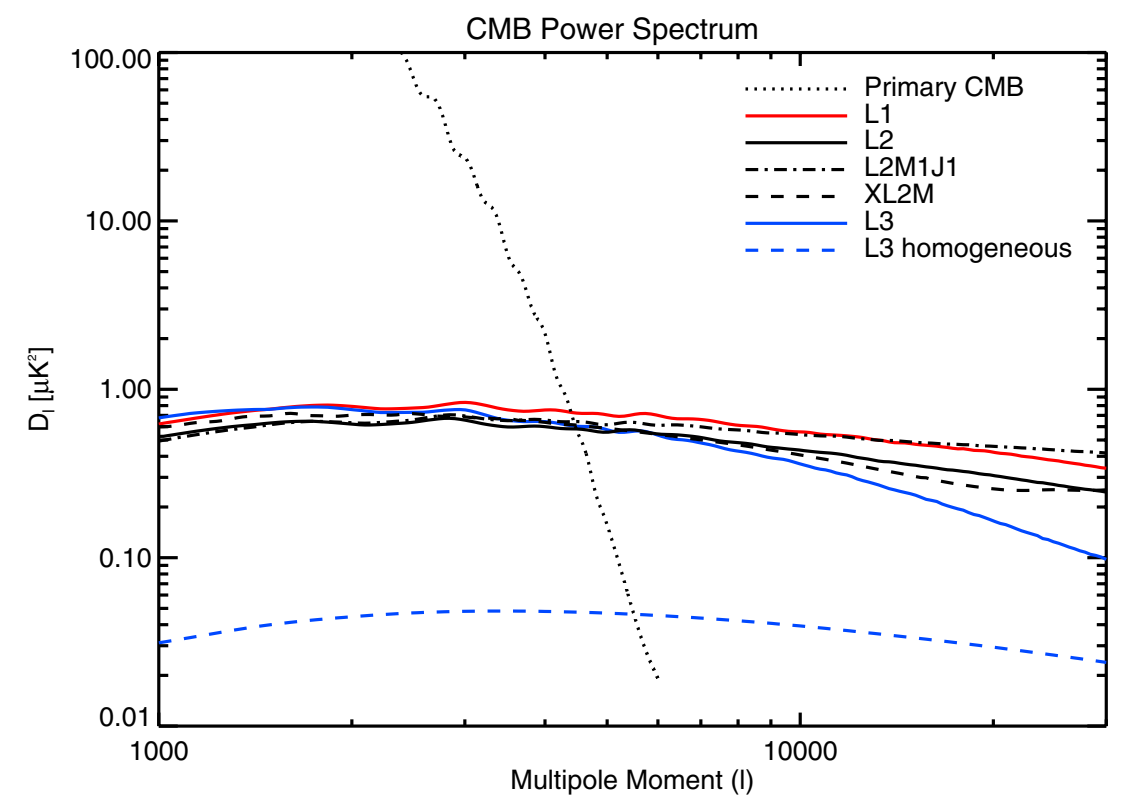

Figure 4. Predicted kSZ power spectra, $D_{l}^{\mathrm{kSZ}}$, from $z>z_{\mathrm{ov}}$ for the models discussed in this work (see Table 1 for the parameters of models). $z_{\mathrm{ov}}=8.3,6.8,6.8,6.8$, and 8.4 for L1, L2, XL2, L2M1J1, and L3, respectively. The box size of L1, L2, L2M1J1, and L3 is $114 h^{-1} \mathrm{Mpc}$, while that of XL2 is $425 h^{-1} \mathrm{Mpc}$. The model parameters of XL2 are the same as those of L2, and thus XL2 provides a useful check of the way we correct for the missing velocity power in $114 h^{-1} \mathrm{Mpc}$ box simulations (see Appendix B for details). The primary CMB power spectrum is also shown.

(A color version of this figure is available in the online journal.)

given by Equation (8). We remind the reader that, on the scales of interest to us in this power spectrum $\left(k \lesssim 1 h \mathrm{Mpc}^{-1}\right)$, the degree of nonlinearity of the underlying density and velocity fields of the IGM is small enough that we can well approximate the kSZ power spectrum for this "homogeneous" ionization case using the assumption of linear perturbations inherent in Equation (8) (see Section 2.2 and the left panel of Figure 1). We use this momentum power spectrum in Equation (6) to obtain the kSZ power spectrum for "L3-homogeneous." Thus, "L3" and "L3-homogeneous" have exactly the same average reionization history, while spatial fluctuations of the ionization fraction are included only in L3. We find that L3 yields an orderof-magnitude larger power spectrum than L3-homogeneous that is consistent with findings in Iliev et al. (2007b; see Figure 4).

In order to see the effect of inhomogeneous reionization on the $\mathrm{kSZ}$ power spectrum in more detail, we show the contribution from a given comoving distance to the kSZ power spectrum at $l=3000, d C_{l=3000}^{\mathrm{kSZ}} / d s$, in Figure 5. While both L3 and L3-homogeneous converge to the same $d C_{l=3000}^{\mathrm{kSZ}} / d s$ after the universe becomes fully ionized, we find a clear enhancement of the power when the ionization fraction is less than unity, $z>z_{\mathrm{ov}}=8.4$. The maximum contribution occurs when the universe is half-ionized. One can see this visually in the middle (L3) and bottom (L3-homogeneous) panels of Figure 5: L3 is clearly more patchy than L3-homogeneous.

The angular scale for $l=3000$ roughly corresponds to the comoving length of $15 \mathrm{~h}^{-1} \mathrm{Mpc}$ during the reionization era $(z \sim 10)$. The contribution to the $\mathrm{kSZ}$ power spectrum continues to grow until the typical comoving size of ionized bubbles reaches $15 h^{-1} \mathrm{Mpc}$. In our models, this occurs when the universe is half-ionized. After this epoch, bubbles grow bigger than $15 h^{-1} \mathrm{Mpc}$, and thus the ionization field is no longer patchy on the scale of $15 h^{-1} \mathrm{Mpc}$. This explains why the contribution to the $\mathrm{kSZ}$ power spectrum at $l=3000$ decreases after the half-ionization epoch. (By the same token, a plot like that for the inhomogeneous case L3 in Figure 5 but for $l>3000$ would look similar, but with the peak shifted to higher $z$ when ionized patches were of smaller scale.)

\subsection{Impact of LMACHs}

How does the presence of LMACHs and self-regulation affect the $\mathrm{kSZ}$ power spectrum? To answer this, we compare L1 and L3, which are mostly similar except that L1 has low-mass halos $\left(10^{8} M_{\odot}<M<2.2 \times 10^{9} M_{\odot}\right)$, with most of them being LMACHs. While they finish reionization at nearly the same redshift, L1 begins ionization earlier due to LMACHs and gives an extended period of low ionization due to self-regulation (see Figure 3).

Figure 4 shows that $\mathrm{L} 1$ and $\mathrm{L} 3$ give similar $\mathrm{kSZ}$ power spectra at $l \lesssim 3000$, while at higher multipoles L1 becomes significantly greater than L3. This is because there are numerous ionized bubbles created by LMACHs at high redshifts, which give significant contributions to the small-scale kSZ power spectrum. Although it would be a challenge for current surveys, future measurements of $D_{l}^{\mathrm{kSZ}}$ with $10 \%$ accuracy over a wide range of multipoles can distinguish between the predictions of $\mathrm{L} 1$ and $\mathrm{L} 3$, shedding light on the roles of LMACHs during reionization.

We compare the contributions from a given comoving distance to the $\mathrm{kSZ}$ power spectrum at $l=3000, d C_{l=3000}^{\mathrm{kSZ}} / d s$, for L1 and L3 in Figure 6. As expected, L1 has larger contributions at higher redshifts $(z \gtrsim 10)$ due to LMACHs. On the other hand, L3 has larger contributions at lower redshifts $(z \lesssim 10)$, as it is more patchy due to the absence of smaller bubbles around LMACHs (see the middle (L3) and bottom (L1) panels of Figure 6). In L1, bubbles around LMACHs do not grow much because of self-regulation.

In the left panel of Figure 7, we show the cumulative contributions to the kSZ power spectrum at $l=3000$ below a given maximum redshift, $z$. This also shows that $\mathrm{L} 1$ receives larger contributions from higher redshifts than L3: 20\% of the total power in $\mathrm{L} 1$ comes from $z>11$, while only a few percent of the total power in L3 comes from $z>11$. Similarly, the 

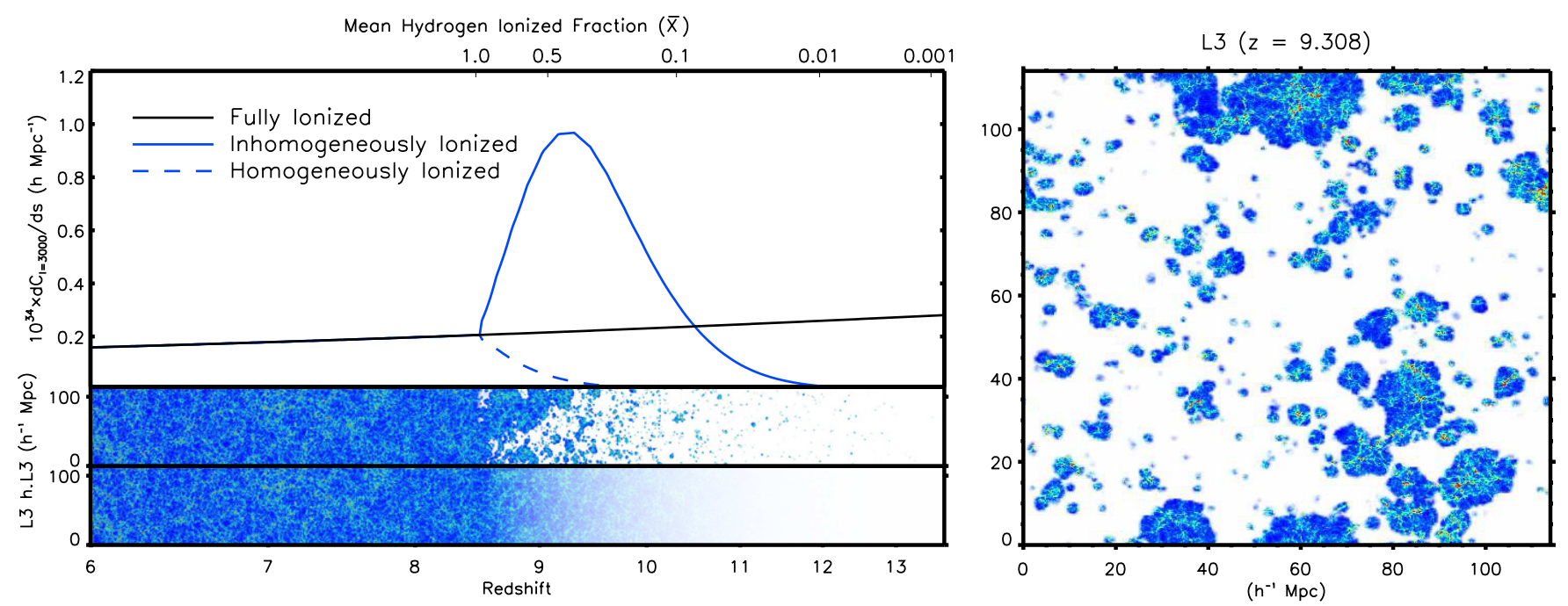

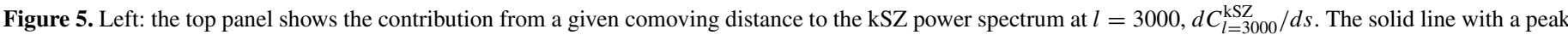

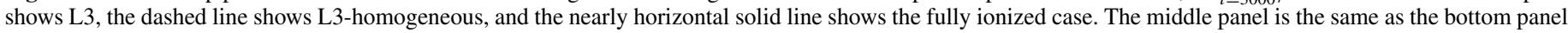

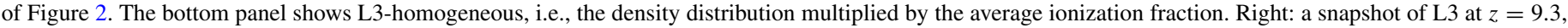
which gives the maximum contribution to the $\mathrm{kSZ}$ power spectrum at $l=3000$.

(A color version of this figure is available in the online journal.)

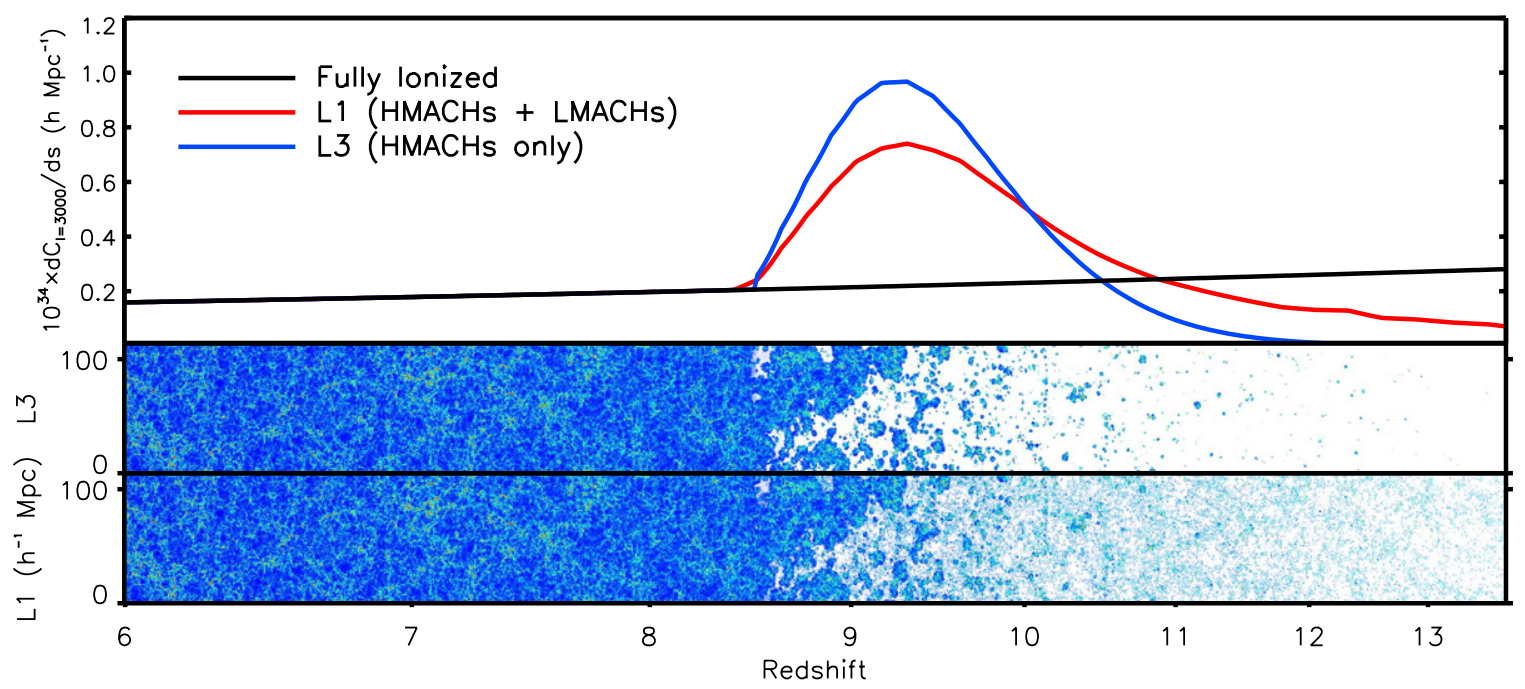

Figure 6. Same as the left panel of Figure 5, but for comparing L1 (bottom panel) and L3 (middle panel). See Table 1 for the parameters of L1 and L3.

(A color version of this figure is available in the online journal.)

right panel of Figure 7 shows that $20 \%$ of the total power in L1 comes from when the ionization fraction is less than 0.25 , which is consistent with the ionization history above $z=11$ shown in Figure 3. This extended tail has important implications for the interpretation of the $\mathrm{kSZ}$ power spectrum, as we will discuss in Section 5.

\subsection{Impact of Mini Halos}

What about MHs? We compare L2 and L2M1J1, which have the same efficiency parameters for HMACHs and LMACHs, but with only L2M1J1 considering MHs. While L2 and L2M1J1 finish reionization at almost the same redshift, L2M1J1 begins ionization much earlier due to $\mathrm{MHs}$ and gives a significantly more extended period of low ionization due to self-regulation (see Figure 3).

Figure 4 shows that L2 and L2M1J1 give similar kSZ power spectra at $l \lesssim 5000$, while at higher multipoles L2M1J1 becomes greater than L2. The reason is the same as that for
L1 versus L3: there are numerous ionized bubbles created by MHs at high redshifts, which contribute to the small-scale kSZ power spectrum.

While L2M1J1 begins reionization much earlier and thus has more contribution from high redshifts to the $\mathrm{kSZ}$ power spectrum, the actual magnitude of the high-redshift contribution is modest. This is because of self-regulation: self-regulation prevents bubbles around MHs from growing, and thus we end up having numerous small bubbles filling space nearly uniformly. This results in a lesser degree of patchiness, and hence a modest contribution to the $\mathrm{kSZ}$ power spectrum at $l=3000$. One can see this visually in the middle (L2M1J1) and bottom (L2) panels of Figure 8. As a result, the situation is similar to that for L1 versus L3: $20 \%$ of the total power at $l=3000$ in L2M1J1 comes from $z>10$, while only $5 \%$ of the total power in L2 comes from $z>10$.

It is interesting that all the models with self-regulation (L1, L2, and L2M1J1) lie on top of each other when the cumulative 

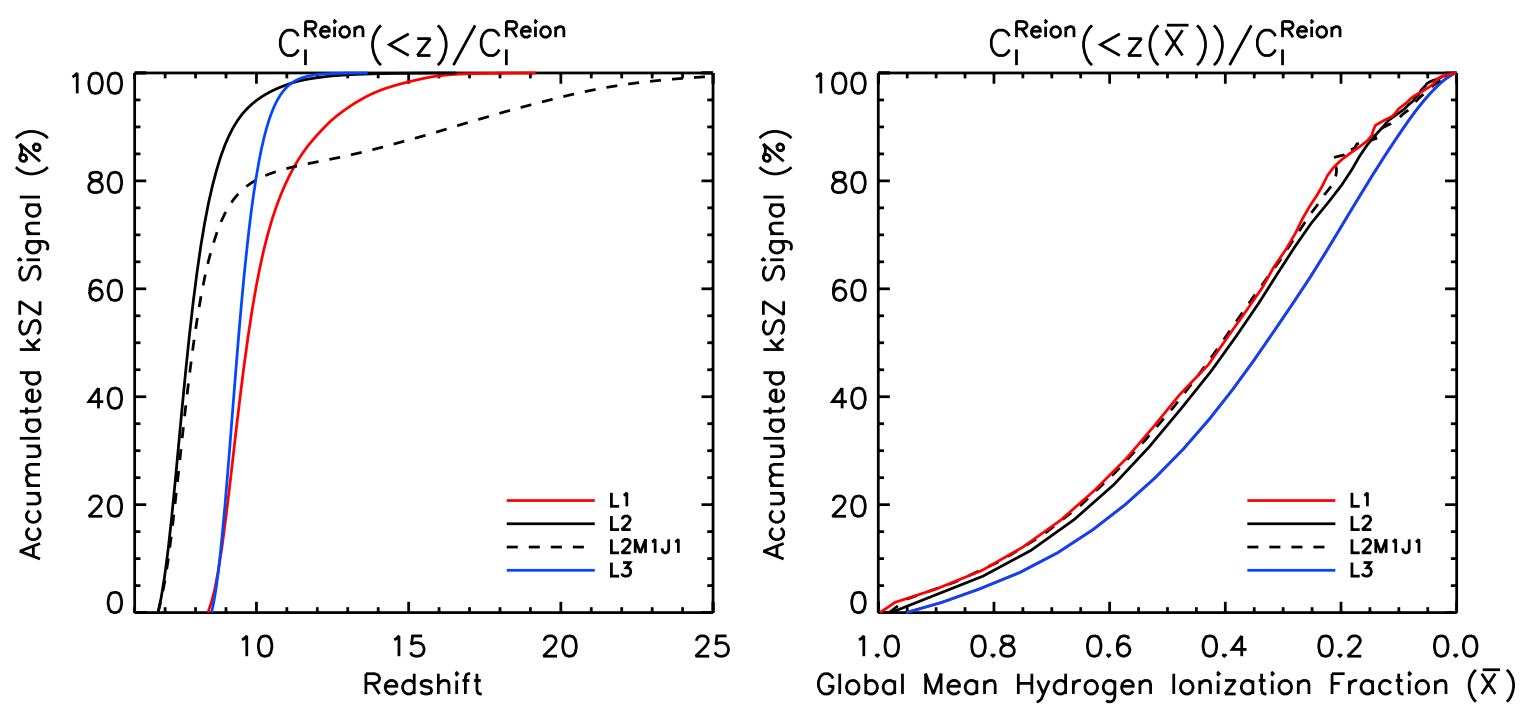

Figure 7. Cumulative reionization $\mathrm{kSZ}$ power spectrum at $l=3000$ as a function of the maximum redshift (left) and the mean ionization fraction (right).

(A color version of this figure is available in the online journal.)

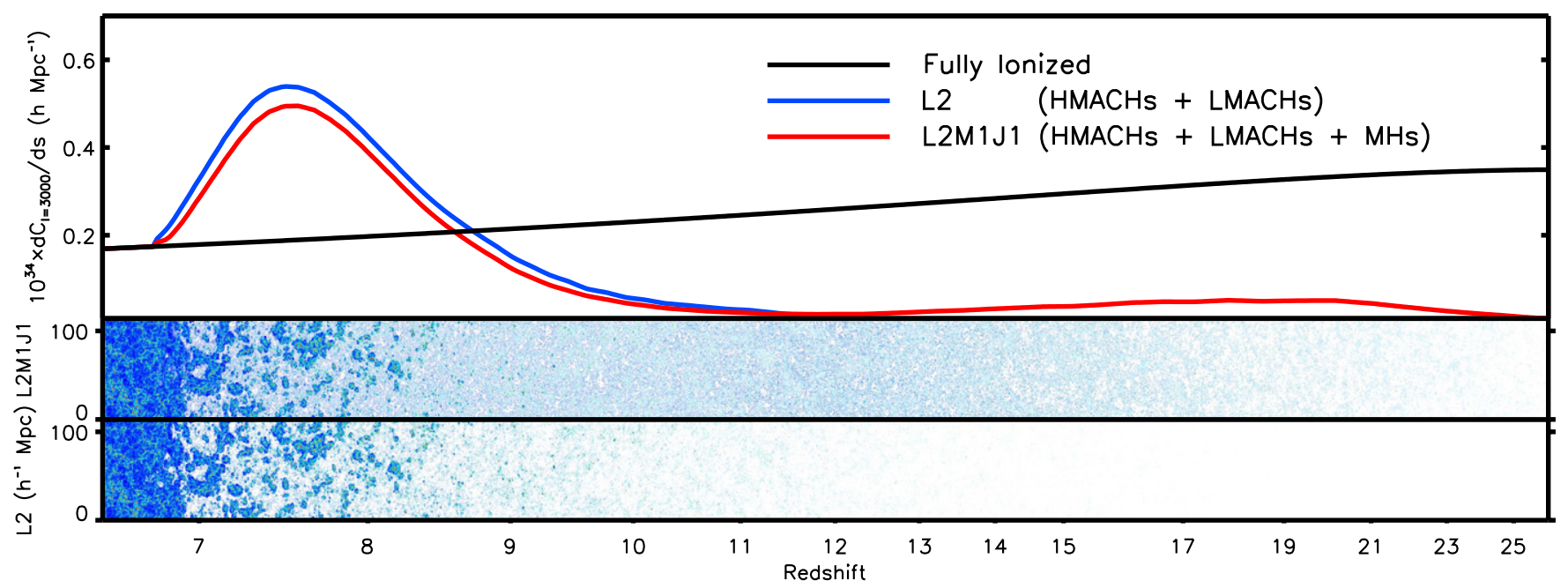

Figure 8. Same as the left panel of Figure 5, but for comparing L2 (bottom panel) and L2M1J1 (middle panel). See Table 1 for the parameters of L2 and L2M1J1.

(A color version of this figure is available in the online journal.)

contribution is shown as a function of the mean ionization fraction (see the right panel of Figure 7), whereas the model that does not have self-regulation (L3) is a clear outlier. Whether this is merely a coincidence or a unique feature of self-regulation is unclear due to the limited number of samples.

\section{SPOT CHECKING THE PREVIOUS CONSTRAINTS ON THE DURATION OF REIONIZATION: MORE EXTENDED HISTORIES CAN GIVE SIMILAR kSZ SIGNALS}

What determines the amplitude of the kSZ power spectrum? Recent studies using semi-numerical reionization models (Zahn et al. 2012; Mesinger et al. 2012; Battaglia et al. 2012a) claim that the amplitude of the $\mathrm{kSZ}$ power spectrum at $l=3000$ can be described by a two-parameter family: the redshift of halfionization, $z_{50 \%}$, and the duration of reionization are defined as $\Delta z \equiv z_{99 \%}-z_{20 \%}$ (Zahn et al. 2012) or $\Delta z \equiv z_{75 \%}-z_{25 \%}$ (Mesinger et al. 2012; Battaglia et al. 2012a). None of these studies included the effects of self-regulated reionization, and thus the reionization histories explored in these studies are roughly symmetric about the epoch of half-ionization.
Figure 2 of Zahn et al. (2012) shows that the kSZ power spectrum at $l=3000$ increases by a factor of two as the duration of reionization increases from $\Delta z=2$ to 4 . Figure 10 of Mesinger et al. (2012) shows that for a half-ionization redshift of $z_{50 \%}=9$, the kSZ power spectrum at $l=3000$ increases by a factor of 1.4 as the duration of reionization increases from $\Delta z=$ 1.3 to 2.6. The former gives a scaling of $D_{l=3000}^{\mathrm{kSZ}} \propto\left(z_{99 \%}-z_{20 \%}\right)$, whereas the latter gives $D_{l=3000}^{\mathrm{kSZ}} \propto\left(z_{75 \%}-z_{25 \%}\right)^{0.5}$ for a fixed half-ionization redshift. More recently, using a new seminumerical method based on a correlation between the smoothed density field and the redshift-of-reionization field found from radiation-hydro simulations of Battaglia et al. (2012b), Battaglia et al. (2012a) calculate the kSZ power spectrum coming from $z>5.5$ and obtain the following scaling relation:

$$
D_{l=3000}^{\mathrm{kSZ}, \mathrm{z} 5.5}=2.02 \mu \mathrm{K}^{2}\left[\left(\frac{1+\bar{z}}{11}\right)-0.12\right]\left(\frac{\Delta z}{1.05}\right)^{0.47},
$$

where $\Delta z=z_{75 \%}-z_{25 \%}$ and $\bar{z}$ is the mean value of the redshiftof-reionization field, which is approximately equal to the half-ionization redshift, $z_{50 \%}$. 
Table 2

Global Reionization History and kSZ Signal

\begin{tabular}{|c|c|c|c|c|c|c|c|c|}
\hline Label & $z_{50 \%}$ & $z 99 \%-z_{20 \%}$ & $z_{75 \%}-z_{25 \%}$ & $z_{\mathrm{ov}}$ & $D_{l=3000}^{\mathrm{kSZ}, \mathrm{z}>5.5}$ & $\begin{array}{l}D_{l=3000}^{\mathrm{kSZ}, \mathrm{z}<\mathrm{z}_{\mathrm{ov}} \mathrm{a}} \\
\end{array}$ & $D_{l=3000}^{\mathrm{kSZ}, \mathrm{z}>\mathrm{z}_{\mathrm{ov}}}$ & $D_{l=3000}^{\mathrm{kSZ} \text {,total }}$ \\
\hline L1 & 9.5 & 3.2 & 2.2 & 8.3 & 1.27 & 1.94 & 0.83 & 2.77 \\
\hline L2 & 7.6 & 2.1 & 1.4 & 6.8 & 0.87 & 1.69 & 0.66 & 2.35 \\
\hline L2M1J1 & 7.7 & 6.5 & 2.1 & 6.8 & 0.90 & 1.69 & 0.69 & 2.38 \\
\hline L3 & 9.1 & 1.3 & 0.9 & 8.4 & 1.20 & 1.96 & 0.75 & 2.71 \\
\hline
\end{tabular}

Note. ${ }^{a}$ From the scaling relation of Shaw et al. (2012).

Our predictions for $D_{l=3000}^{\mathrm{kSZ}}$ are summarized in Table 2 . Among the models we have explored in this paper, L3 (which contains only HMACHs and does not have self-regulation) closely matches the scenarios explored in the above studies. Using $z_{50 \%}=9.1$ and $z_{75 \%}-z_{25 \%}=0.9$ that we find for L3, Equation (12) gives $D_{l=3000}^{\mathrm{kSZ}, \mathrm{z}>5.5}=1.5 \mu \mathrm{K}^{2}$. This is in reasonable agreement with our result, ${ }^{10} D_{l=3000}^{\mathrm{kSZ}, \mathrm{z}>5.5}=1.2 \mu \mathrm{K}^{2}$.

However, the above formula significantly overestimates the amplitude of the kSZ power spectrum for L1: Equation (12) gives $D_{l=3000}^{\mathrm{kSZ}, \mathrm{z}>5.5}=2.4 \mu \mathrm{K}^{2}$, whereas we find $D_{l=3000}^{\mathrm{kSZ}, \mathrm{z}>5}=$ $1.3 \mu \mathrm{K}^{2}$. In other words, despite the fact that L1 has a significantly more extended duration of reionization than L3 (by a factor of more than two), $z_{75 \%}-z_{25 \%}=2.2$, the amplitude of the kSZ power spectrum increases only by $8 \%$. Similarly, Equation (12) gives $D_{l=3000}^{\mathrm{kSZ}, \mathrm{z}>5.5}=1.5$ and $1.9 \mu \mathrm{K}^{2}$ for $\mathrm{L} 2$ and L2M1J1, respectively, whereas we find $0.9 \mu \mathrm{K}^{2}$ for both cases. Therefore, we conclude that Equation (12) is valid only for simple scenarios where the reionization history is roughly symmetric about the half-ionization redshift, but is invalid when self-regulation is included. Similar conclusions apply to Zahn et al. (2012) and Mesinger et al. (2012).

Our results show that self-regulation makes the duration of reionization significantly more extended without changing the amplitude of the kSZ power spectrum very much. In other words, an extended period of low-level ionization in $z>z_{50 \%}$ does not contribute much to the $\mathrm{kSZ}$ power spectrum at $l=3000$.

\section{CONCLUSION}

In this paper, using the state-of-the-art reionization simulations incorporating the effects of self-regulated reionization (Iliev et al. 2012; Ahn et al. 2012), we have computed the power spectrum of the $\mathrm{kSZ}$ effect from the EOR. Unlike the previous work which created maps and computed two-dimensional Fourier transforms from the maps, we have computed the $\mathrm{kSZ}$ power spectrum from a line-of-sight integral of the transverse momentum power spectrum of ionized gas. We present a method to statistically correct for the missing velocity power in Appendix B, and verify the accuracy of our method by comparing the results from large- $\left(425 \mathrm{Mpc} h^{-1}\right)$ and small-box (114 Mpc $h^{-1}$ ) simulations.

We find that the kSZ power spectrum is a sensitive probe of the patchiness of reionization: patchiness increases the amplitude of the $\mathrm{kSZ}$ power spectrum by an order of magnitude. The maximum contribution occurs when the angular sizes of ionized bubbles are close to those corresponding to a given multipole.

While inclusion of small-mass halos such as LMACHs and MHs makes the beginning of reionization earlier, self-regulation

\footnotetext{
10 In order to compute $D_{l=3000}^{\mathrm{kSZ}, \mathrm{z}>5.5}$, we calculate the contribution from $z$

between 5.5 and $z_{\mathrm{ov}}$ using the fully ionized formula, $P_{q_{\perp}}=P_{q_{\perp}}^{\mathrm{OV}}$, and add it to $D_{l=3000}^{\mathrm{kSZ}, \mathrm{z}>\mathrm{z}_{\mathrm{ov}}}$, shown in the seventh column of Table 2 .
}

significantly slows down the progress of reionization (Iliev et al. 2007a, 2012; Ahn et al. 2012). This results in an extended period of low-level ionization before more massive HMACHs dominate and finish reionization. We find that such an extended period of low-level ionization does not, however, make much of a contribution to the $\mathrm{kSZ}$ power spectrum at $l=3000: D_{l=3000}^{\mathrm{kSZ}}$ changes only by $\sim 10 \%$ despite the fact that the duration of reionization increases by a factor of more than two.

Our results qualitatively change the conclusions reached by the previous work which did not include self-regulation. Recent works (Zahn et al. 2012; Mesinger et al. 2012; Battaglia et al. 2012a) assume that $D_{l=3000}^{\mathrm{kSZ}}$ can be adequately parameterized by the redshift of half-ionization, $z_{50 \%}$, and the duration of reionization, $\Delta z$. While our result for the simplest model of reionization without self-regulation (L3) agrees with the scaling formula of Battaglia et al. (2012a; Equation (12)), our results for the models with self-regulation do not agree with it: specifically, the amplitude of the $\mathrm{kSZ}$ effect is no longer correlated well with the duration of the reionization. This is because selfregulation gives an extended period of low-level reionization only for $z>z_{20 \%}$, while the simple models adopted by these other treatments have a roughly symmetric reionization history of about $z=z_{50 \%}$, for which a longer duration thus implies a longer period of the patchy state with a significant ionization across $z=z_{50 \%}$. Therefore, a more accurate scaling formula is required to take into account the asymmetric reionization history typical of self-regulated reionization.

Going beyond $l=3000$, we find that LMACHs and MHs do have a considerable impact on the $\mathrm{kSZ}$ power spectrum on smaller angular scales. For example, $D_{l=10000}^{\mathrm{kSZ}}$ is boosted by $60 \%$ and $25 \%$ when LMACHs and MHs are included, respectively. Even though measurements of the $\mathrm{kSZ}$ power spectrum at $l>3000$ would be a challenge for the moment due to contamination by extragalactic point sources and $\mathrm{tSZ}$, future multiwavelength observations may allow us to determine the $\mathrm{kSZ}$ power spectrum from the EOR over a wide range of multipoles. Such measurements will provide us with valuable additional information on the nature of the ionizing sources and the history of reionization.

How do our calculations compare with these current observational constraints? In order to obtain the total $\mathrm{kSZ}$ signal from both reionization and post-reionization contributions, we take the "cooling and star formation" post-reionization model of Shaw et al. (2012) that approximately incorporates the Jeans filtering of $P_{q_{\perp}}$ due to shock heating in halos and in the IGM. The post-reionization $\mathrm{kSZ}$ signal computed from their scaling relation and the total $\mathrm{kSZ}$ signal (i.e., the sum of our reionization calculation and their post-reionization calculation) are shown in the sixth and seventh columns of Table 2, respectively. We find that all of our predictions are consistent with the 95\% CL upper bound on the total signal from SPT, $D_{l=3000}^{\mathrm{kSZ} \text {,total }}<2.8 \mu \mathrm{K}^{2}$ (Reichardt et al. 2012). Therefore, we conclude that the current 
data are consistent with our understanding of the physics of reionization.

We are indebted to D. Munshi for pointing out typos now corrected in the intermediate steps in Appendix A. K.A. was supported in part by NRF grants funded by the Korean government MEST (Nos. 2012R1A1A1014646, 2012M4A2026720). I.T.I. was supported by The Southeast Physics Network (SEPNet) and the Science and Technology Facilities Council grants ST/F002858/1 and ST/I000976/1. This study was supported in part by the Swedish Research Council grant 2009-4088, U.S. NSF grants AST-0708176 and AST-1009799, NASA grants NNX07AH09G, NNG04G177G, and NNX11AE09G, and Chandra grant SAO TM8-9009X. The authors acknowledge the TeraGrid and the Texas Advanced Computing Center (TACC) at The University of Texas at Austin (URL: http://www.tacc.utexas.edu), and the Swedish National Infrastructure for Computing (SNIC) resources at HPC2N (Umeå, Sweden) for providing HPC and visualization resources that have contributed to the results reported within this paper.

\section{APPENDIX A}

\section{DERIVATION OF THE POWER SPECTRUM OF THE kSZ EFFECT}

\section{A.1. Suppression of Longitudinal Modes}

An important observation of the nature of $\mathrm{kSZ}$ is that it is given by the transverse (vector-mode or spin-1) momentum field, and the longitudinal contribution is suppressed. To show this, we Fourier transform Equation (5):

$$
\frac{\Delta T}{T}(\hat{\gamma})=-\frac{\sigma_{T} n_{e, 0}}{c} \int \frac{d s}{a(s)^{2}} e^{-\tau} \int \frac{d^{3} k}{(2 \pi)^{3}}[\hat{\gamma} \cdot \tilde{\mathbf{q}}(\mathbf{k}, s)] e^{-i \mathbf{k} \cdot(s \hat{\gamma})} .
$$

Decomposing the momentum vector in Fourier space, $\tilde{\mathbf{q}}$, into the longitudinal component, $\tilde{q}_{\|} \equiv \tilde{\mathbf{q}} \cdot \hat{k}$, and the transverse component, $\tilde{q}_{\perp} \equiv|\tilde{\mathbf{q}}-\hat{k}(\tilde{\mathbf{q}} \cdot \hat{k})|$, we obtain

$$
\frac{\Delta T}{T}(\hat{\gamma})=-\frac{\sigma_{T} n_{e, 0}}{c} \int \frac{d s}{a(s)^{2}} e^{-\tau} \int \frac{d^{3} k}{(2 \pi)^{3}}\left[x \tilde{q}_{\|}(\mathbf{k}, s)+\cos \left(\phi_{\hat{q}}-\phi_{\hat{\gamma}}\right)\left(1-x^{2}\right)^{1 / 2} \tilde{q}_{\perp}(\mathbf{k}, s)\right] e^{-i k s x},
$$

where $x \equiv \hat{k} \cdot \hat{\gamma}$, and $\phi_{\hat{q}}$ and $\phi_{\hat{\gamma}}$ are the angles that $\mathbf{k}$ makes with $\tilde{\mathbf{q}}$ and $\hat{\gamma}$, respectively.

If the factor $e^{i k s x}$ oscillates much more rapidly than the other quantities, then the integral over $s$ will be small due to cancellation. If we recall that $a(s), \tau(s)$, and $\tilde{\mathbf{q}}$ all vary over the Hubble length scale, then $k x$ should be much smaller than $H / c$ in order to avoid the cancellation. Namely, either the wavelength should be longer than the Hubble length, i.e., $k \lesssim H / c$, or the mode should be nearly perpendicular to the line-of-sight direction, i.e., $x \approx 0$. The former does not contribute much because the amplitude of such a long-wavelength mode is small. Thus, only the modes that are perpendicular to the line-of-sight direction, $x \approx 0$, have a chance to contribute to the $\mathrm{kSZ}$ signal.

However, in this configuration, the longitudinal component of the momentum field is also perpendicular to the line of sight, and vanishes when taken a dot-product with the line of sight, i.e., $x \tilde{q}_{\|} \approx 0$. Therefore, only the transverse mode survives in the integral, giving

$$
\frac{\Delta T}{T}(\hat{\gamma})=-\frac{\sigma_{T} n_{e, 0}}{c} \int \frac{d s}{a(s)^{2}} e^{-\tau} \int \frac{d^{3} k}{(2 \pi)^{3}} \cos \left(\phi_{\hat{q}}-\phi_{\hat{\gamma}}\right)\left(1-x^{2}\right)^{1 / 2} \tilde{q}_{\perp}(\mathbf{k}, s) e^{-i k s x} .
$$

\section{A.2. Angular Power Spectrum}

Here, we follow steps similar to those in Chapter 7.3 of Weinberg (2008) to derive the angular power spectrum of CMB fluctuations induced by the $\mathrm{kSZ}$ effect.

Spherical-harmonic decomposition of Equation (A3) gives

$$
\begin{aligned}
a_{l m} & =\int d^{2} \hat{\gamma} Y_{l}^{m *}(\hat{\gamma}) \frac{\Delta T}{T}(\hat{\gamma}) \\
& =-\frac{\sigma_{T} n_{e, 0}}{c} \int d^{2} \hat{\gamma} Y_{l}^{m *}(\hat{\gamma}) \int \frac{d s}{a(s)^{2}} e^{-\tau} \int \frac{d^{3} k}{(2 \pi)^{3}} \cos \left(\phi_{\hat{q}}-\phi_{\hat{\gamma}}\right)\left(1-x^{2}\right)^{1 / 2} \tilde{q}_{\perp}(\mathbf{k}, s) e^{-i k s x} \\
& \equiv-\frac{\sigma_{T} n_{e, 0}}{c} \int \frac{d^{3} k}{(2 \pi)^{3}} f_{l m}(\mathbf{k})
\end{aligned}
$$

where

$$
\begin{aligned}
f_{l m}(\mathbf{k}) \equiv & \int d^{2} \hat{\gamma} Y_{l}^{m *}(\hat{\gamma}) \int \frac{d s}{a(s)^{2}} e^{-\tau} \cos \left(\phi_{\hat{q}}-\phi_{\hat{\gamma}}\right)\left(1-x^{2}\right)^{1 / 2} \tilde{q}_{\perp}(\mathbf{k}, s) e^{-i k s x} \\
= & \int d^{2} \hat{\gamma} Y_{l}^{m *}(\hat{\gamma}) \int \frac{d s}{a(s)^{2}} e^{-\tau} \cos \left(\phi_{\hat{q}}-\phi_{\hat{\gamma}}\right)\left(1-x^{2}\right)^{1 / 2} \tilde{q}_{\perp}(\mathbf{k}, s) \\
& \times 4 \pi \sum_{L M}(-i)^{L} j_{L}(k s) Y_{L}^{M}(\hat{\gamma}) Y_{L}^{M *}(\hat{k}) .
\end{aligned}
$$


We first choose a convenient coordinate system in which the $z$-direction lies on that of the mode vector, i.e., $\hat{k}=\hat{z}$, and the azimuthal direction is the same as the direction of the momentum vector, i.e., $\phi_{\hat{q}}=0$. In this case, $Y_{L}^{M *}(\hat{k})$ simplifies to $Y_{L}^{M *}(\hat{z})=\delta_{M 0} \sqrt{\frac{2 L+1}{4 \pi}}$, giving

$$
\begin{aligned}
f_{l m}(k \hat{z}) & =\sqrt{4 \pi} \int \frac{d s}{a(s)^{2}} e^{-\tau} \tilde{q}_{\perp}(\mathbf{k}, s) \sum_{L}(-i)^{L} \sqrt{2 L+1} j_{L}(k s) \int d^{2} \hat{\gamma} Y_{L}^{0}(\hat{\gamma}) \cos \phi \sin \theta Y_{l}^{m *}(\hat{\gamma}) \\
& =\sqrt{\frac{8 \pi^{2}}{3}} \int \frac{d s}{a(s)^{2}} e^{-\tau} \tilde{q}_{\perp}(\mathbf{k}, s) \sum_{L}(-i)^{L} \sqrt{2 L+1} j_{L}(k s) \int d^{2} \hat{\gamma} Y_{L}^{0}(\hat{\gamma})\left[Y_{1}^{-1}(\hat{\gamma})-Y_{1}^{1}(\hat{\gamma})\right] Y_{l}^{m *}(\hat{\gamma}),
\end{aligned}
$$

where $\theta$ and $\phi=\phi_{\hat{\gamma}}$ determine the line-of-sight vector as $\hat{\gamma}=(\cos \theta \sin \phi, \sin \theta \sin \phi, \cos \phi)$.

The integral over $\hat{\gamma}$ can be computed using

$$
\int d^{2} \hat{\gamma} Y_{L}^{M}(\hat{\gamma}) Y_{\Lambda}^{\mu}(\hat{\gamma}) Y_{l}^{m *}(\hat{\gamma})=\sqrt{\frac{(2 \Lambda+1)(2 l+1)}{4 \pi(2 L+1)}} C_{l \Lambda}(L, M ; m,-\mu) C_{l \Lambda}(L, 0 ; 0,0) \delta_{M, m-\mu},
$$

where $C_{l \Lambda}(L, M ; m, \mu)$ is the Clebsch-Gordan coefficient for adding the angular momentum quantum numbers $(l, m)$ and $(\Lambda, \mu)$ and for forming $(L, M)$. In our case, we have

$$
\left.f_{l, m= \pm 1}(k \hat{z})=\sqrt{2 \pi(2 l+1)} \int \frac{d s}{a(s)^{2}} e^{-\tau} \tilde{q}_{\perp}(\mathbf{k}, s) \sum_{L}(-i)^{L} j_{L}(k s) \times\left[ \pm C_{l 1}(L, 0 ; \pm 1, \mp 1) C_{l 1}(L, 0 ; 0,0)\right)\right] .
$$

Thus, the relevant coefficients are

$$
\begin{aligned}
C_{l 1}(l+1,0 ; 0,0) & =\sqrt{\frac{l+1}{2 l+1}}, \quad C_{l 1}(l+1,0 ; \pm 1, \mp 1)=\sqrt{\frac{l}{2(2 l+1)}}, \\
C_{l 1}(l, 0 ; 0,0) & =0, \quad C_{l 1}(l-1,0 ; 0,0)=\sqrt{\frac{l}{2 l+1}}, \quad C_{l 1}(l-1,0 ; \pm 1, \mp 1)=\sqrt{\frac{l+1}{2(2 l+1)}} .
\end{aligned}
$$

Putting these together gives

$$
\begin{aligned}
f_{l, m= \pm 1}(k \hat{z}) & =(-i)^{l+1} \sqrt{\frac{\pi l(l+1)}{2 l+1}} \int \frac{d s}{a(s)^{2}} e^{-\tau} \tilde{q}_{\perp}(\mathbf{k}, s)\left[j_{l+1}(k s)+j_{l-1}(k s)\right] \\
& =(-i)^{l+1} \sqrt{\pi l(l+1)(2 l+1)} \int \frac{d s}{a(s)^{2}} e^{-\tau} \tilde{q}_{\perp}(\mathbf{k}, s) \frac{j_{l}(k s)}{k s} .
\end{aligned}
$$

Now, we get back to the observer's frame by applying the standard rotation operator, $S(\hat{q})$, that takes the $z$-direction into $\hat{k}$. This gives

$$
f_{l m}(\mathbf{k})=\sum_{m^{\prime}= \pm 1} D_{m, m^{\prime}}^{l}(S(\hat{k})) f_{l m^{\prime}}(k \hat{z})
$$

where $D_{m m^{\prime}}^{l}=\left\langle l, m^{\prime}|S| l, m\right\rangle$ is the matrix representation of the finite rotation of an initial state $(l, m)$ into a final state $\left(l, m^{\prime}\right)$. We obtain

$$
a_{l m}=-\frac{\sigma_{T} n_{e, 0}}{c} \int \frac{d^{3} k}{(2 \pi)^{3}} \sum_{m^{\prime}= \pm 1} D_{m, m^{\prime}}^{l}(S(\hat{k}))(-i)^{l+1} \sqrt{\pi l(l+1)(2 l+1)} \int \frac{d s}{a(s)^{2}} e^{-\tau} \tilde{q}_{\perp}(\mathbf{k}, s) \frac{j_{l}(k s)}{k s} .
$$

Finally, we calculate the angular power spectrum from $\left\langle a_{l m} a_{l^{\prime} m^{\prime}}^{*}\right\rangle=C_{l} \delta_{l l^{\prime}} \delta_{m m^{\prime}}$ and obtain

$$
C_{l}=\frac{l(l+1)}{\pi}\left(\frac{\sigma_{T} n_{e, 0}}{c}\right)^{2} \int \frac{d s}{a(s)^{2}} e^{-\tau(s)} \int \frac{d s^{\prime}}{a\left(s^{\prime}\right)^{2}} e^{-\tau\left(s^{\prime}\right)} \int k^{2} d k \frac{j_{l}(k s)}{k s} \frac{j_{l}\left(k s^{\prime}\right)}{k s^{\prime}} P_{q_{\perp}}(k, s),
$$

where $P_{q_{\perp}}$ is the power spectrum of $\tilde{\mathbf{q}}_{\perp}$ defined by $(2 \pi)^{3} P_{q_{\perp}}(k) \delta^{D}\left(\mathbf{k}-\mathbf{k}^{\prime}\right)=\left\langle\tilde{\mathbf{q}}_{\perp}(\mathbf{k}) \tilde{\mathbf{q}}_{\perp}^{*}\left(\mathbf{k}^{\prime}\right)\right\rangle$. Here, we have used the identity

$$
\int d^{2} \hat{k} D_{m, \pm 1}^{l}(S(\hat{k})) D_{m^{\prime}, \pm 1}^{l^{\prime} *}(S(\hat{k}))=\frac{4 \pi}{2 l+1} \delta_{m m^{\prime}} \delta_{l l^{\prime}} .
$$

The integral over $k$ can be performed with Limber's approximation: when a function $g(k, s)$ varies much more slowly than the spherical Bessel function, one can approximate the integral as

$$
\int k^{2} d k j_{l}(k s) j_{l}\left(k s^{\prime}\right) g(k, s) \approx \frac{\pi}{2} \frac{\delta^{D}\left(s-s^{\prime}\right)}{s^{2}} g\left(k=\frac{l}{s}, s\right) .
$$


With this approximation, we finally obtain the desired formula for the kSZ power spectrum:

$$
C_{l}=\left(\frac{\sigma_{T} n_{e, 0}}{c}\right)^{2} \int \frac{d s}{s^{2} a(s)^{4}} e^{-2 \tau(s)} \frac{P_{q_{\perp}}(k=l / s, s)}{2} .
$$

This is Equation (6).

\section{APPENDIX B}

\section{CORRECTING FOR THE MISSING POWER IN SIMULATIONS}

The transverse momentum power spectrum at a given wavenumber, $P_{q_{\perp}}(k)$, receives contributions from the density and velocity auto/cross power spectra at various wavenumbers via Equation (9). As a result, $P_{q_{\perp}}$ computed from a simulation with a finite box suffers from a loss of power due to the lack of modes whose wavelength is greater than the size of the box (Iliev et al. 2007b).

The missing power arises because we do not have $P_{\chi(1+\delta), \chi(1+\delta)}(k), P_{v v}(k)$, or $P_{\chi(1+\delta), v}(k)$ for $k<k_{\text {box }} \equiv 2 \pi / l_{\text {box }}$, where $l_{\text {box }}$ is the size of the box. In Equation (9), this leads to the missing contributions in $\left|\mathbf{k}^{\prime}\right|<k_{\mathrm{box}}$ and $\left|\mathbf{k}-\mathbf{k}^{\prime}\right|<k_{\mathrm{box}}$. Estimating and correcting for the missing power thus requires knowledge of the large-scale limit of $P_{\chi(1+\delta), \chi(1+\delta)}, P_{v v}$, and $P_{\chi(1+\delta), v}$.

For the homogeneous reionization case, it is straightforward to recover the missing power, as the large-scale limits of $P_{v v}$, $P_{\chi(1+\delta), \chi(1+\delta)}\left(=\bar{\chi}^{2} P_{\delta \delta}\right)$, and $P_{\chi(1+\delta), v}\left(=\bar{\chi} P_{\delta v}\right)$ are precisely known by the cosmological linear perturbation theory. Using $P_{\delta \delta}$ from the linear theory and the linear relation, $P_{v v}=(\dot{a} f / k)^{2} P_{\delta \delta}$, we find that the missing-power-corrected momentum power spectrum from the $N$-body simulation agrees precisely with the expected OV spectrum (see Figure 1). Note that most of the missing power comes from $P_{\delta \delta}\left(\left|\mathbf{k}-\mathbf{k}^{\prime}\right|\right) P_{v v}\left(k^{\prime}\right)$ in $k^{\prime}<k_{\text {box }}$ because of the relation, $\mathbf{v}(\mathbf{k}) \propto \delta(\mathbf{k}) / k$, in the large-scale limit.

For the inhomogeneous reionization case, we do not have a precise way to calculate the ionized density power, $P_{\chi(1+\delta), \chi(1+\delta)}$, in the large-scale limit; however, we expect that the density field and the ionization field are reasonably flat at scales larger than the box size, and correct for the missing bulk velocity of the box. Therefore, we expect that the term $P_{\chi(1+\delta), \chi(1+\delta)}\left(\left|\mathbf{k}-\mathbf{k}^{\prime}\right|\right) P_{v v}\left(k^{\prime}\right)$ in $k^{\prime}<k_{\text {box }}$ captures most of the missing power, as we have seen from the homogeneous reionization case above. With this approximation, the missing power in the inhomogeneously ionized regime is given by

$$
P_{q_{\perp}}^{\text {Missing }}(k, z)=\int_{k<k_{\mathrm{box}}} \frac{d^{3} k^{\prime}}{(2 \pi)^{3}}\left(1-\mu^{\prime 2}\right) P_{\chi(1+\delta), \chi(1+\delta)}\left(\left|\mathbf{k}-\mathbf{k}^{\prime}\right|\right) P_{v v}\left(k^{\prime}\right) .
$$

In order to check the accuracy of Equation (B1), we compare the missing-power-corrected momentum power spectrum from the box of $114 h^{-1}$ Mpc (black solid line; denoted as L2) with that from a larger box of $425 h^{-1}$ Mpc (black dashed line; XL2) in Figure 4. We find a very good agreement between the two, confirming the robustness of our correction for the missing power.

\section{REFERENCES}

Ahn, K., Iliev, I. T., Shapiro, P. R., et al. 2012, ApJL, 756, L16

Battaglia, N., Natarajan, A., Trac, H., Cen, R., \& Loeb, A. 2012a, arXiv: 1211.2832

Battaglia, N., Trac, H., Cen, R., \& Loeb, A. 2012b, arXiv:1211.2821

Crawford, T. M., Schaffer, K. K., Bhattacharya, S., et al. 2013, arXiv:1303.3535

Dunkley, J., Calabrese, E., Sievers, J., et al. 2013, arXiv:1301.0776

Fernandez, E. R., Iliev, I. T., Komatsu, E., \& Shapiro, P. R. 2012, ApJ, 750,20

Fernandez, E. R., Komatsu, E., Iliev, I. T., \& Shapiro, P. R. 2010, ApJ, 710,1089

Gnedin, N. Y., \& Jaffe, A. H. 2001, ApJ, 551, 3

Gruzinov, A., \& Hu, W. 1998, ApJ, 508, 435

Harnois-Deraps, J., Pen, U.-L., Iliev, I. T., et al. 2012, arXiv:1208.5098

Iliev, I. T., Mellema, G., Pen, U.-L., Bond, J. R., \& Shapiro, P. R. 2008, MNRAS, 384,863

Iliev, I. T., Mellema, G., Shapiro, P. R., \& Pen, U.-L. 2007a, MNRAS, 376, 534

Iliev, I. T., Mellema, G., Shapiro, P. R., et al. 2012, MNRAS, 423, 2222

Iliev, I. T., Pen, U.-L., Bond, J. R., Mellema, G., \& Shapiro, P. R. 2007b, ApJ, 660,933

Iliev, I. T., \& Shapiro, P. R. 2001, MNRAS, 325, 468

Jaffe, A. H., \& Kamionkowski, M. 1998, PhRvD, 58, 043001

Komatsu, E., Dunkley, J., Nolta, M. R., et al. 2009, ApJS, 180, 330

Ma, C.-P., \& Fry, J. N. 2002, PhRvL, 88, 211301
McQuinn, M., Furlanetto, S. R., Hernquist, L., Zahn, O., \& Zaldarriaga, M. 2005, ApJ, 630, 643

Mellema, G., Iliev, I. T., Alvarez, M. A., \& Shapiro, P. R. 2006, NewA, 11, 374 Mesinger, A., McQuinn, M., \& Spergel, D. N. 2012, MNRAS, 422, 1403

Refregier, A., Komatsu, E., Spergel, D. N., \& Pen, U.-L. 2000, PhRvD, 61,123001

Reichardt, C. L., Shaw, L., Zahn, O., et al. 2012, ApJ, 755, 70

Salvaterra, R., Ciardi, B., Ferrara, A., \& Baccigalupi, C. 2005, MNRAS, 360,1063

Santos, M. G., Cooray, A., Haiman, Z., Knox, L., \& Ma, C.-P. 2003, ApJ, 598,756

Shapiro, P. R., Giroux, M. L., \& Babul, A. 1994, ApJ, 427, 25

Shapiro, P. R., Iliev, I. T., Mellema, G., et al. 2012, in AIP Conf. Ser. 1480, First Stars IV, ed. M. Umemura \& K. Omukai (Melville, NY: AIP), 248

Shaw, L. D., Rudd, D. H., \& Nagai, D. 2012, ApJ, 756, 15

Sunyaev, R. A., \& Zel'dovich, I. B. 1980, MNRAS, 190, 413

Tseliakhovich, D., \& Hirata, C. 2010, PhRvD, 82, 083520

Vishniac, E. T. 1987, ApJ, 322, 597

Weinberg, S. 2008, Cosmology (Oxford, U.K.: Oxford Univ. Press),

Zahn, O., Mesinger, A., McQuinn, M., et al. 2011, MNRAS, 414, 727

Zahn, O., Reichardt, C. L., Shaw, L., et al. 2012, ApJ, 756, 65

Zahn, O., Zaldarriaga, M., Hernquist, L., \& McQuinn, M. 2005, ApJ, 630, 657

Zel'dovich, Y. B., \& Sunyaev, R. A. 1969, Ap\&SS, 4, 301 